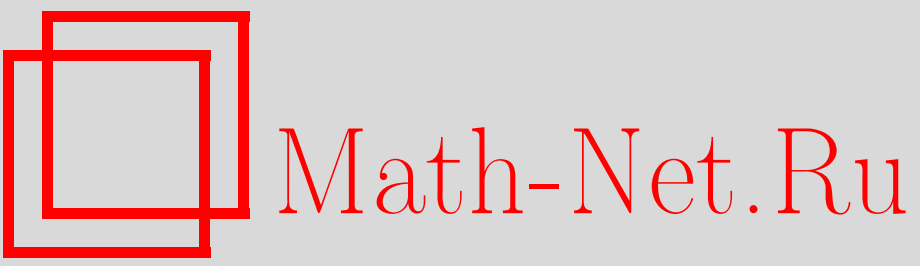

M. А. Соловьев, РCT, спин и статистика и аналитический волновой фронт, TMФ, 1999, том 121, номер 1, 139-164

DOI: https://doi.org/10.4213/tmf802

Использование Общероссийского математического портала Math-Net.Ru подразумевает, что вы прочитали и согласны с пользовательским соглашением

http://www.mathnet.ru/rus/agreement

Параметры загрузки:

IP : 35.173 .137 .237

26 апреля 2023 г., 12:49:05 


\author{
ТЕОРЕТИЧЕСКАЯ \\ И МАТЕМАТИЧЕСКАЯ \\ ФИЗИКА \\ Том 121, № 1 \\ октябрь, 1999
}

(C) 1999 г.

М. А. Соловьев*

\title{
РСТ, СПИН И СТАТИСТИКА И АНАЛИТИЧЕСКИЙ ВОЛНОВОЙ ФРОНТ
}

Излагается новый, более общий вывод теорем о связи спина со статистикой и $P C T$ инвариантности. Он основан на использовании понятия аналитического волнового фронта (ультра)распределения и в отличие от обычного охватывает нелокальные квантовые поля. В качестве функциональной области определения полей используются пробные функции с компактным носителем в импульсном представлении. Тем самым допускается сколь угодно высокая сингулярность вакуумных средних в координатном пространстве. Условие локальной коммутативности заменяется требованием асимптотической коммутативности, которое развивает обобщения аксиомы микропричинности, предлагавшиеся ранее.

\section{1. ВВЕДЕНИЕ}

В данной статье исследуется, насколько сушественны локальность взаимодействия и аксиома микропричинности для вывода основных экспериментально проверяемых следствий обшей теории квантовых полей: связи спина со статистикой и $P C T$-симметрии. Оказывается, оба следствия теории остаются в силе при замене локальной коммутативности более близким к макропричинности условием, которое мы называем асимптотической коммутативностью и которое на интуитивном уровне означает экспоненциальное убывание коммутаторов наблюдаемых полей с порядком, равным единице, и максимальным типом (или, для краткости, убывание быстрее любой линейной экспоненты) при пространственноподобном разделении аргументов. Точное определение этого условия дано ниже, оно усиливает формулировку этого условия, данную в работе [1], где указано его соотношение с обобшениями аксиомы локальности, предлагавшимися другими авторами. При отказе от микропричинности естественно отказаться и от ограничений на высокоэнергетическое (ультрафиолетовое) поведение амплитуд, которые из нее вытекают (см. [2, § 9.1.Д; $3, \S$ VII.4]). Мы считаем это поведение произвольным и тем самым допускаем сколь угодно сингулярную зависимость поля от координат пространства-времени. В этом случае обычный вывод связи спина со статистикой и $P C T$-симметрии [2-4], основанный на свойствах аналитичности вакуумных средних

\footnotetext{
1) Статья написана по заказу Редколлегии.
}

\footnotetext{
* Физический институт им. П. Н. Лебедева РАН, Москва, Россия
} 
в $x$-пространстве, неприменим, ранее предлагалось заменить его построением оболочек голоморфности в $p$-пространстве [5-7]. В работах [8, 9] замечено, что решение проблемы достигается путем использования понятия аналитического волнового фронта распределения. В настоящей работе излагается новый вывод теорем о связи спина со статистикой и РCT-симметрии на основе такого подхода. Мы стремимся к максимальной общности рассмотрения и поэтому используем в качестве функциональной области определения полей пространства Гельфанда-Шилова $S_{\alpha}^{0}$ вместо пространства $S^{0}$ (фурье-образа пространства $\mathcal{D}$, состоящего из функций с компактным носителем), использовавшегося в [5-9]. Иначе говоря, поля считаются не распределениями, а ультрараспределениями в импульсном представлении. Важную роль в доказательстве играют недавние результаты теории аналитических функционалов, полученные в работах [10-13], главным из которых является существование у функционалов класса $S_{\alpha}^{\prime 0}$ однозначно определенных минимальных несущих конусов ${ }^{2)}$. Применение структурных теорем, касаюшихся свойств такого квазиносителя, позволяет обращаться с высокосингулярными обобщенными функциями столь же легко, как с обычными распределениями Шварца.

Квантовая теория высокосингулярных взаимодействий является наиболее разработанным направлением нелокальной теории поля, обзор основных результатов можно найти в [14-17]. Особо отметим, что в его рамках развита последовательная теория асимптотических состояний и частиц $[14,18]$, включающая вывод ограничений на амплитуды рассеяния [14]. Использование высокосингулярных нелокальных формфакторов оказалось эффективным при феноменологическом описании сильных взаимодействий [16], интересны также возможные связи этого направления теории поля с теорией струн $[1,19]$.

Статья организована следуюшим образом. Раздел 2 содержит краткое изложение упомянутых выше структурных теорем. В разделе 3 устанавливается связь между аналитическим волновым фронтом ультрараспределения и несущим конусом его преобразования Фурье и доказывается теорема единственности, которая показывает несовместимость определенных свойств сосредоточенности в $x$ - и $p$-пространствах и является ключевой в нашем подходе. Условие асимптотической коммутативности формулируется в разделе 4 как условие на матричные элементы поля и затем переводится на язык вакуумных средних. В разделе 5 исследуется специфика инвариантной ультрафиолетовой регуляризации лоренц-ковариантных функционалов класса $S_{\alpha}^{\prime 0}$. В разделе 6 эта регуляризация используется для выяснения роли точек Йоста в нелокальной теории поля. Раздел 7 содержит доказательства аналогов всех основных пунктов классического вывода теоремы о связи спина со статистикой [2-4], начиная с леммы Дел'Антонио и кончая теоремой Араки о существовании преобразования Клейна, приводяшего поля к нормальным перестановочным соотношениям, для теории поля, подчиненной условию асимптотической коммутативности. В разделе 8 вводится условие слабой асимптотической коммутативности и доказана его эквивалентность $P C T$-инвариантности. Раздел 9 посвяшен заключительным замечаниям.

\footnotetext{
2) Здесь и ниже штрих обозначает сопряженное пространство, состоящее из линейных непрерывных функционалов.
} 


\section{2. НЕСУЩИЕ КОНУСЫ АНАЛИТИЧЕСКИХ ФУНКЦИОНАЛОВ. ОСНОВНЫЕ ТЕОРЕМЫ}

В стандартном изложении аксиоматического подхода [2-4] поля предполагаются операторнозначными распределениями умеренного роста, определенными на пробных функциях из пространства Шварца $S$. Главное основание для этого состоит в том, что в теории возмушений сингулярности всегда имеют конечный порядок. Однако, когда речь идет о точных решениях или об обших следствиях теории, такое предположение становится излишне ограничительным [20]. Для расширения рамок формализма удобно использовать пространства $S_{\alpha}^{\beta}$ Гельфанда-Шилова [21]. Напомним, что $S_{\alpha}^{\beta}\left(\mathbb{R}^{n}\right)$ представляет собой объединение (точнее, индуктивньй предел) семейства пространств $S_{\alpha, a}^{\beta, b}$ $(a, b>0)$, состояших из бесконечно дифференцируемых функций на $\mathbb{R}^{n}$, для которых конечна норма

$$
\|f\|_{a, b}=\sup _{x \in \mathbb{R}^{n}} \sup _{k, q \in \mathbb{Z}_{+}^{n}} \frac{\left|x^{k} \partial^{q} f(x)\right|}{a^{|k|} b^{|q|} k^{\alpha k} q^{\beta q}} .
$$

Ясно, что индекс $\alpha$ определяет скорость роста функционалов из сопряженного пространства $S_{\alpha}^{\prime \beta}$, а индекс $\beta$ - их сингулярность, т.е. в контексте квантовой теории поля они определяют инфракрасное и ультрафиолетовое поведение полей. В соответствии со сказанным во введении мы полагаем $\beta=0$. Пространство $S_{\alpha}^{0}$ нетривиально лишь при $\alpha>1$, и всюду в дальнейшем это условие предполагается выполненным. Фурье-преобразованное пространство $S_{0}^{\alpha}$ состоит из функций с компактным носителем, поэтому локальные свойства обобшенных функций из $S_{0}^{\prime \alpha}$, которые называют ультрараспределениями Румье класса $\left\{k^{\alpha k}\right\}$, практически те же, что и у распределений Шварца, и их носители определяются обычным способом, с помошью разложения единицы. Напротив, само пространство $S_{\alpha}^{0}$ состоит из целых аналитических функций, т.е. в $x$-представлении элементы сопряженного пространства являются аналитическими функционалами, для которых понятие носителя не имеет смысла. Тем не менее функционалы этого класса сохраняют своего рода угловую локализуемость, которая может быть описана посредством следуюшей конструкции [10]. Каждому открытому конусу в $U \subset \mathbb{R}^{n}$ можно сопоставить пространство $S_{\alpha}^{0}(U)$, определяемое аналогично $S_{\alpha}^{0}$, но с той разницей, что в аналоге формулы (1) точная верхняя грань должна вычисляться по $x \in U$. Функция $f \in S_{\alpha, a}^{0, b}(U)$ допускает аналитическое продолжение в $\mathbb{C}^{n}$, удовлетворяющее оценке

$$
|f(x+i y)| \leqslant C \exp \left\{-\left|\frac{x}{a^{\prime}}\right|^{\frac{1}{\alpha}}+b^{\prime} d(x, U)+b^{\prime}|y|\right\}
$$

где $d(\cdot, U)$ - расстояние от точки до конуса $U, a^{\prime}, b^{\prime}$ отличаются от $a, b$ на числовой множитель, зависящий от выбора нормы ${ }^{3)}$ в $\mathbb{R}^{n}$. Это отличие несущественно, ибо по индексам $a, b$ берется объединение, и оценку (2) тоже можно положить в основу определения $S_{\alpha}^{0}(U)$. Пространства над конусами обладают теми же удобными для функционального анализа свойствами, что и исходные $S_{\alpha}^{0},-$ они являются полными, бочечными,

${ }^{3)} \mathrm{B}$ дальнейшем мы, как правило, считаем ее евклидовой. 
рефлексивными, ядерными и монтелевскими (см. [17]). Пространство $S_{\alpha}^{0}$, очевидно, является линейным подпространством каждого $S_{\alpha}^{0}(U)$, а $S_{\alpha}^{\prime 0}(U)$ можно отождествить с линейным подпространством в $S_{\alpha}^{\prime 0}$ благодаря следующей теореме.

ТЕОрема 1 (о плотности). Пространство $S_{\alpha}^{0}$ плотно в $S_{\alpha}^{0}(U)$ для любого открытого конуса $U \subset \mathbb{R}^{n}$.

Мы называем замкнутьй конус $K \subset \mathbb{R}^{n}$ несущим конусом аналитического функционала $v \in S_{\alpha}^{\prime 0}$ и говорим, что этот функционал сосредоточен на $K$ (что соответствует английскому термину carried by $K$ ), если $v$ допускает непрерывное продолжение на каждое пространство $S_{\alpha}^{0}(U)$, где $U \supset K \backslash\{0\}$. Это свойство непрерывности равносильно принадлежности $v \in S_{\alpha}^{\prime 0}(K)$, где пространство $S_{\alpha}^{0}(K)$ определяется как объединение указанных $S_{\alpha}^{0}(U)$ и снабжается топологией индуктивного предела. Отдельного определения требует пространство $S_{\alpha}^{0}(\{0\})$, соответствующее вырожденному конусу $\{0\}$ - началу координат. Оно состоит из целых функций, удовлетворяющих неравенству $|f(z)| \leqslant C \exp (b|z|)$, где $z=x+i y$, а константы $C, b$ зависят от $f$. Из оценки поведения пробных функций видно, что сосредоточенность $v$ на $K$ можно интуитивно представлять себе и как свойство убывания этого функционала в дополнительном к $K$ конусе быстрее любой линейной экспоненты.

ТЕорема 2 (о квазилокализуемости). Если функиионал $v \in S_{\alpha}^{\prime 0}$ сосредоточен на замкнутых конусах $K_{1}, K_{2}$, то он сосредоточен и на их пересечении.

Как следствие, в силу обычных соображений компактности, сушествует наименьший замкнутый конус $K$ такой, что $v \in S_{\alpha}^{\prime 0}(K)$. Мы называем его квазиносителем $v$.

ТЕОрема 3 (о разложении). Каждый функционал $v \in S_{\alpha}^{\prime 0}$, сосредоточенный на обгединении двух замкнутых конусов $K_{1}, K_{2}$, допускает разложсение вида $v=v_{1}+$ $v_{2}$, где $v_{j} \in S_{\alpha}^{\prime 0}\left(K_{j}\right), \quad j=1,2$.

Классы аналитических функционалов со свойствами, описываемыми теоремами 1-3, естественно называть квазираспределениями.

Если конус $K$ является острым, т.е. сопряженный ему конус $K^{*}=\{\eta: \eta x \geqslant 0, \forall x \in$ $K\}$ имеет непустую внутренность, то $e^{i \zeta x} \in S_{\alpha}^{0}(K)$ при всех $\operatorname{Im} \zeta$ из этой внутренности, и тогда для всякого $v \in S_{\alpha}^{\prime 0}(K)$ определено преобразование Лапласа

$$
\check{\mathbf{v}}(\zeta)=(2 \pi)^{-\frac{n}{2}}\left(v, e^{i \zeta x}\right) .
$$

Теорема 4 (типа Пэли-Винера-Шварца). Пусть функиионал $v \in S_{\alpha}^{\prime 0}$ сосредоточен на замкнутом остром конусе $K$. Его преобразование Лапласа г̌ аналитично в трубчатой области $\mathbb{R}^{n}+i V$, где $V$ - внутренность конуса $K^{*}$, и удовлетворяет оиенке

$$
|\check{\mathbf{v}}(\zeta)| \leqslant C_{\varepsilon, R}\left(V^{\prime}\right) \exp \left\{\varepsilon|\operatorname{Im} \zeta|^{-\frac{1}{\alpha-1}}\right\} \quad\left(\operatorname{Im} \zeta \in V^{\prime},|\zeta| \leqslant R\right)
$$

при всех $\varepsilon, R>0$ и для любого конуса $V^{\prime}$ такого, что $\bar{V}^{\prime} \backslash\{0\} \subset V$. Если $\operatorname{Im} \zeta \rightarrow 0$ внутри фиксированного $V^{\prime}$, то $\check{\mathbf{v}}(\zeta)$ стремится $к$ фурье-образу $\check{v}$ функиионала $v$ по 
топологии $S_{0}^{\prime \alpha}$. Если конус $K \kappa$ тому же выпукльй, то преобразование Лапласа устанавливает алгебрачческий и топологический изоморфизм межсу пространством $S_{\alpha}^{\prime 0}(K)$ и пространством аналитических в $\mathbb{R}^{n}+i V$ функиий, удовлетворяющих ограничению вида (3).

Теорема 1 доказана в работе [12], теоремы 2 и 3 - в [10], а теорема 4 - в [11, 12] с помошью $L^{2}$-оценок Хёрмандера для решений системы неоднородных уравнений Коши-Римана и сопряженного ей уравнения. Отметим, что аналогичные теоремы справедливы и для функциональных классов $S_{\alpha}^{\prime \beta}, 0<\beta<1$, причем устанавливаются даже более простым способом [1], однако именно пространство $S_{\alpha}^{0}$ представляет особый интерес, ибо при его использовании не накладывается никаких ограничений на ультрафиолетовое поведение полей и в этом смысле оно универсально. Стоит подчеркнуть, что термины несущий конус и квазиноситель можно использовать без конкретизации индекса $\alpha$ (что вполне естественно, ибо он характеризует поведение на бесконечности, а не локальные свойства). Действительно, принадлежность $v \in S_{\alpha}^{\prime 0}(K)$ очевидным образом влечет $v \in S_{\alpha^{\prime}}^{\prime 0}(K)$ для всех $\alpha^{\prime}<\alpha$. С другой стороны, в работе [13] доказана следуюшая теорема.

Tеорема 5. Ecлu $v \in S_{\alpha}^{\prime 0} \cap S_{\alpha^{\prime}}^{\prime 0}(K)$, əде $\alpha^{\prime}<\alpha$, mo $v \in S_{\alpha}^{\prime 0}(K)$.

Аналогичное утверждение для $S_{\alpha}^{\prime \beta}$, где $\beta \neq 0$, прямо следует из теоремы 3 работы [1], а при $\beta=0$ снова приходится использовать оценки Хёрмандера. Ниже будет полезен еще один простой факт, который является частным случаем теоремы 2 работы [1].

Лемма 1 (о свертке). Для каждого $v \in S_{\alpha}^{\prime 0}(K)$ и всякой пробной функции $f \in S_{\alpha}^{0}$

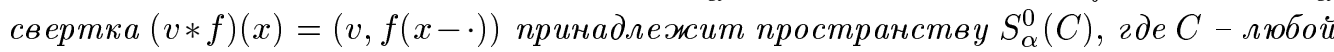
открытый конус такой, что $\bar{C} \backslash\{0\} \subset \complement K$. При этом отображсние $S_{\alpha}^{0} \rightarrow S_{\alpha}^{0}(C)$ : $f \rightarrow v * f$ непрерьвно.

Это почти очевидно, ибо сдвиг пробной функции внутрь дополнительного конуса СK означает удаление от несушего конуса $K$.

\section{3. АНАЛИТИЧЕСКИЙ ВОЛНОВОЙ ФРОНТ И КВАЗИНОСИТЕЛЬ}

Хорошо известно (см., например, $[22, \S$ I.2.b]), что ультрараспределения вложены в пространство гиперфункций с сохранением носителей. Поэтому, если мы имеем дело с аналитическим волновым фронтом $W F_{A}(u)$ ультрараспределения $u \in S_{0}^{\prime \alpha}\left(\mathbb{R}^{n}\right)$, то можно использовать обшие факты [23], установленные для гиперфункций. Напомним, что $W F_{A}(u)$ является замкнутым подмножеством в $\mathbb{R}^{n} \times\left(\mathbb{R}^{n} \backslash\{0\}\right)$, коническим относительно второй переменной. Его проекция на первый сомножитель совпадает с наименьшим замкнутым множеством, вне которого $u$ аналитично, обозначаемым sing $\operatorname{supp}_{A} u$, a ассоциируемый с точкой $p \in \operatorname{sing} \operatorname{supp}_{A} u$ конус составляют те направления "плохого" поведения фурье-образа $u$ на бесконечности, которые ответственны за неаналитичность в этой точке. 
Лемма 2. Если и $\in S_{0}^{\prime \alpha}\left(\mathbb{R}^{n}\right)$ и если замкнутый конус $K \subset \mathbb{R}^{n}$ является несущим для преобразования Фурье $\hat{u}, m^{4)}$

$$
W F_{A}(u) \subset \mathbb{R}^{n} \times(K \backslash\{0\}) .
$$

ДОКАЗАТЕЛЬСТвО. Покроем $K$ конечным числом замкнутых, острых, выпуклых конусов $K_{j}$ и, используя теорему 3 , произведем разложение

$$
\hat{u}=\sum \hat{u}_{j}, \quad \hat{u}_{j} \in S_{\alpha}^{\prime 0}\left(K_{j}\right) .
$$

Согласно теореме 4 разложение (5) порождает представление $u$ в виде суммы граничных значений функций $\mathbf{u}_{j}(\zeta)$, аналитических в трубчатых областях $\mathbb{R}^{n}+i V_{j}$, где $V_{j}$ внутренность сопряженного к $K_{j}$ конуса $K_{j}^{*}$. Эти граничные значения совпадают с граничными значениями в смысле гиперфункций (см., например, [24, теорема 11.5]), и согласно теореме 9.3.4 из книги [23] справедливо включение

$$
W F_{A}(u) \subset \mathbb{R}^{n} \times\left(\cup K_{j}^{* *} \backslash\{0\}\right),
$$

где $K_{j}^{* *}=K_{j}$, поскольку $K_{j}$ замкнуты и выпуклы. Измельчая покрытие и стягивая его к $K$, получаем включение (4).

ЗАмечАнИЕ. Лемма 2 усиливает лемму 8.4.17 книги [23], согласно которой для распределения умеренного роста $u \in S^{\prime}$ соблюдается включение $W F_{A}(u) \subset \mathbb{R}^{n} \times(L \backslash\{0\})$, где $L-$ предельный конус для supp $\hat{u}$ на бесконечности. Отметим в этой связи, что конус $L$ заведомо является несушим для ограничения $\hat{u} \mid S_{\alpha}^{0}$.

Действительно, этот конус по определению состоит из пределов последовательностей $t_{\nu} x_{\nu}$, где $x_{\nu} \in \operatorname{supp} \hat{u}$ и $0<t_{\nu} \rightarrow 0$. Если $L=\{0\}$, то носитель $\hat{u}$ ограничен и $\left(\hat{u}, f_{\nu}\right) \rightarrow 0$ для любой последовательности $f_{\nu}$, сходящейся к нулю в $S_{\alpha}^{0}(\{0\})$, ибо в силу неравенства Коши эта сходимость влечет равномерную сходимость $f_{\nu}$ к нулю на компактах вместе со всеми производными. Если $L \neq\{0\}$, то для всякого открытого конуса $U \supset L \backslash\{0\}$ множество supp $\hat{u} \backslash U$ компактно. Тогда сходимость последовательности $f_{\nu}$ к нулю в $S_{\alpha}^{0}(U)$ влечет сходимость к нулю по каждой из норм $\sup _{U \cup B}\left|x^{k} \partial^{q} f(x)\right|$, где $B$ - любая ограниченная окрестность этого компакта, и мы снова заключаем, что $\left(\hat{u}, f_{\nu}\right) \rightarrow 0$.

Теорема 6. Пусть $u \in S_{0}^{\prime \alpha}\left(\mathbb{R}^{n}\right)$ - нетривиальное ультрараспределение с носителем в остром конусе $V$. Тогда лишь все пространство $\mathbb{R}^{n}$ может служить несущим конусом его преобразования Фурье $\hat{u}$.

${ }^{4)}$ Отметим, что оператор $u \rightarrow \hat{u}$ сопряжен преобразованию пробных функций

$$
f(x) \rightarrow(2 \pi)^{-\frac{n}{2}} \int e^{-i p x} f(x) d x
$$

со знаком минус в экспоненте, в отличие от участвовавшего в теореме 4 обратного ему оператора $v \rightarrow \check{v}$, определение которого отличается знаком. 
ДоказАтельство. Сначала будем считать, что $0 \in \operatorname{supp} u$. Тогда любой вектор из конуса $-K^{*} \backslash\{0\}$ служит внешней нормалью к носителю в точке 0. Согласно теореме Касивары [23, теорема 9.6.6, следствие 9.6.8] все ненулевые элементы линейной оболочки множества внешних нормалей принадлежат $W F_{A}(u)_{p=0}$. Поскольку конус $V$ острый, внутренность $V^{*}$ непуста, и эта линейная оболочка покрывает $\mathbb{R}^{n}$. Значит, согласно лемме 2 несуший конус $\hat{u}$ должен совпадать с $\mathbb{R}^{n}$.

Пусть теперь $0 \notin \operatorname{supp} u$. Предположим, что существует открытый конус $U$ такой, что $\bar{U} \neq \mathbb{R}^{n}$ и $\hat{u} \in S_{\alpha}^{\prime 0}(U)$. Рассмотрим ряд из “сжатых" ультрараспределений

$$
\sum_{\nu=1}^{\infty} c_{\nu} u_{\nu}
$$

где

$$
\left(u_{\nu}, g(p)\right) \stackrel{\text { def }}{=} \frac{\left(u, g\left(\frac{p}{\nu}\right)\right)}{\nu^{n}} .
$$

Пусть $\|\cdot\|_{U, a, b}^{\prime}-$ норма сопряженного к $S_{\alpha, a}^{0, b}(U)$ банахова пространства. Если

$$
0<c_{\nu}<\frac{1}{\nu^{2}\left\|\hat{u}_{\nu}\right\|_{U, \nu, \nu}^{\prime}}
$$

то ряд $\sum c_{\nu} \hat{u}_{\nu}$ сходится в каждом пространстве $S_{\alpha, a}^{\prime 0, b}(U)$ и тем более ряд $(7)$ сходится в $S_{0}^{\prime \alpha}$. Обозначим его сумму через $\tilde{u}$. Коэффициенты $c_{\nu}$ нетрудно выбрать так, что носитель $\tilde{u}$ будет содержать точку 0 . Действительно, пусть $p_{0}-$ ближайшая к 0 точка множества $\operatorname{supp} u$. Не ограничивая обшности, можно считать, что $\left|p_{0}\right|=1$. Для каждого $\mu=1,2, \ldots$ найдется функция $g_{\mu} \in S_{0}^{\alpha}$ с носителем в шаре $\left|p-p_{0} / \mu\right|<(1 / 2)(1 / \mu-$ $1 /(\mu+1))$ такая, что $\left(u_{\mu}, g_{\mu}\right)=1$. Отметим, что supp $u_{\nu} \cap \operatorname{supp} g_{\mu}=\varnothing$ при $\nu<\mu$. Зададим коэффициенты $c_{\nu}$ последовательно, подчиняя их помимо (8) условиям

$$
c_{\nu}\left|\left(u_{\nu}, g_{\mu}\right)\right| \leqslant \frac{a_{\mu}}{2^{\nu}} \text { при } \mu<\nu
$$

Тогда при любом $\mu$

$$
\left(\tilde{u}, g_{\mu}\right)=a_{\mu}+\sum_{\nu>\mu} c_{\nu}\left(u_{\nu}, g_{\mu}\right) \neq 0
$$

Тем самым $0 \in \operatorname{supp} \tilde{u}$, и мы вернулись к уже рассмотренной ситуации, что завершает доказательство.

Отметим, что теореме 6 можно придать форму теоремы единственности, в которой она и будет использоваться далее. А именно, если относительно ультрараспределения $u \in S_{0}^{\prime \alpha}\left(\mathbb{R}^{n}\right)$ известно, что его носитель лежит в остром конусе, а несущий конус $\hat{u}$ отличен от $\mathbb{R}^{n}$, то $u=0$.

6 Теоретическая и математическая физика, т. 121, № 1, 1999 г. 


\section{4. АСИМПТОТИЧЕСКАЯ КОММУТАТИВНОСТЬ}

Мы будем рассматривать конечную совокупность полей $\left\{\phi_{\iota}\right\}, \iota=1, \ldots, I$, являющихся операторнозначными обобщенными функциями над пространством $S_{\alpha}^{0}\left(\mathbb{R}^{4}\right)$, $\alpha>1$, и преобразуюшихся по конечномерным неприводимым представлениям собственной группы Лоренца $L_{+}^{\uparrow}$ или ее накрывающей $S L(2, \mathbb{C})$. Считаем выполненными все обычные предположения вайтмановского аксиоматического подхода [2-4], за исключением аксиомы локальной коммутативности, которую невозможно сформулировать в терминах пробных функций, принадлежащих $S_{\alpha}^{0}$, поскольку они являются целыми аналитическими в координатном представлении. Как обычно, обозначаем через $D_{0}$ минимальную инвариантную и предполагаемую плотной общую область определения полевых операторов в гильбертовом пространстве состояний $\mathcal{H}$, т.е. векторное подпространство, порождаемое вакуумным состоянием $\Psi_{0}$ и всевозможными векторами вида

$$
\phi_{\iota_{1} \ell_{1}}\left(f_{1}\right) \ldots \phi_{\iota_{n} \ell_{n}}\left(f_{n}\right) \Psi_{0}, \quad n=1,2, \ldots
$$

где $f_{k} \in S_{\alpha}^{0}\left(\mathbb{R}^{4}\right)$ и $\ell_{k}-$ лоренщевы индексы.

ОПРЕДЕЛЕниЕ 1 . Полевые компоненты $\phi_{\iota \ell}$ и $\phi_{\iota^{\prime} \ell^{\prime}}$ называем асимптотически коммутируюшими (антикоммутируюшими) при пространственноподобном разделении аргументов, если для любых векторов $\Phi, \Psi \in D_{0}$ аналитический функционал

$$
\left\langle\Phi,\left[\phi_{\iota \ell}(x), \phi_{\iota^{\prime} \ell^{\prime}}\left(x^{\prime}\right)\right]_{(+)} \Psi\right\rangle
$$

сосредоточен на замкнутом конусе $\overline{\mathcal{V}}=\left\{\left(x, x^{\prime}\right) \in \mathbb{R}^{8}:\left(x-x^{\prime}\right)^{2} \geqslant 0\right\}$.

Отметим, что матричный элемент (9) можно считать обобщенной функцией на $S_{\alpha}^{0}\left(\mathbb{R}^{8}\right)$ благодаря установленному в [12] совпадению этого пространства с $\tau_{i}$-пополненным тензорным произведением $S_{\alpha}^{0}\left(\mathbb{R}^{4}\right) \widehat{\otimes}_{i} S_{\alpha}^{0}\left(\mathbb{R}^{4}\right)$, сопряженное пространство к которому канонически изоморфно пространству билинейных раздельно непрерьвных функционалов на $S_{\alpha}^{0}\left(\mathbb{R}^{4}\right) \times S_{\alpha}^{0}\left(\mathbb{R}^{4}\right)$ по определению индуктивной топологии $\tau_{i}$. Такое совпадение имеет место и в случае нескольких тензорных сомножителей, поэтому в полной аналогии со стандартной схемой [2-4] $n$-точечные вакуумные средние полей однозначно определяют вайтмановские обобшенные функции $\mathcal{W}_{\iota_{1} \ell_{1}, \ldots, \iota_{n} \ell_{n}} \in S_{\alpha}^{\prime 0}\left(\mathbb{R}^{4 n}\right)$, с которыми мы их в дальнейшем отождествляем. Очевидным образом определяются выражения

$$
\int \phi_{\iota_{1} \ell_{1}}\left(x_{1}\right) \ldots \phi_{\iota_{n} \ell_{n}}\left(x_{n}\right) f\left(x_{1}, \ldots, x_{n}\right) d x_{1} \ldots d x_{n} \Psi_{0}, \quad n=1,2, \ldots,
$$

где $f \in S_{\alpha}^{0}\left(\mathbb{R}^{4 n}\right)$. Именно, вектор (10) представляет собой предел последовательности состояний

$$
\Psi_{N}=\sum_{\nu=1}^{N} \phi_{\iota_{1} \ell_{1}}\left(f_{1}^{\nu}\right) \ldots \phi_{\iota_{n} \ell_{n}}\left(f_{n}^{\nu}\right) \Psi_{0}
$$

где

$$
\sum_{\nu=1}^{N} f_{1}^{\nu}\left(x_{1}\right) \ldots f_{n}^{\nu}\left(x_{n}\right)=f_{N} \in S_{\alpha}^{0}\left(\mathbb{R}^{4}\right)^{\otimes n}
$$


и $f_{N} \rightarrow f$ в $S_{\alpha}^{0}\left(\mathbb{R}^{4 n}\right)$. Векторы вида (10) порождают подпространство $D_{1} \supset D_{0}$, на которое каждый оператор $\phi_{\iota}(f)$ может быть продолжен по непрерывности. Подчеркнем, что полевые операторы образуют неприводимую систему. Это устанавливается тем же способом, что и в обычной вайтмановской формулировке [2]. Пусть $M(f)=$ $\phi_{\iota_{1} \ell_{1}}\left(f_{1}\right) \ldots \phi_{\iota_{n} \ell_{n}}\left(f_{n}\right)$ - моном по полевым компонентам, а $T(r)$ - оператор трансляции пространства-времени. Если ограниченный оператор $B$ в $\mathcal{H}$ коммутирует в слабом смысле со всеми операторами поля, то

$$
\left\langle M(f)^{*} \Psi_{0}, B \Psi_{0}\right\rangle=\left\langle\Psi_{0}, B M(f) \Psi_{0}\right\rangle
$$

Заменяя здесь $M(f)$ на моном $T(r) M(f) T^{-1}(r)=M(f(\cdot-r))$ и учитывая трансляционную инвариантность вакуума, получаем

$$
\left\langle M(f)^{*} \Psi_{0}, T^{-1}(r) B \Psi_{0}\right\rangle=\left\langle B^{*} \Psi_{0}, T(r) M(f) \Psi_{0}\right\rangle .
$$

Эта функция вектора $r$ является гладкой и ограничена константой $\left\|B^{*} \Psi_{0}\right\|\left\|M(f) \Psi_{0}\right\|$, поэтому преобразование Фурье этой функции принадлежит классу распределений умеренного роста. В силу спектрального условия носитель фурье-образа левой части равенства (11) лежит в замкнутом верхнем световом конусе $\overline{\mathbb{V}}_{+}$, а правой части - в конусе $\overline{\mathbb{V}}_{-}$. Значит, этим носителем служит $\{0\}$, а функция (11) является ограниченным полиномом, т.е. константой. Ввиду произвольности монома $M(f)$ и плотности $D_{0}$ в $\mathcal{H}$ независимость левой части формулы (11) от $r$ означает инвариантность вектора $B \Psi_{0}$ относительно пространственно-временных трансляший. Отсюда следует, что $B \Psi_{0}=\lambda \Psi_{0}$, поскольку вакуум предполагается единственным инвариантным состоянием. Записывая теперь

$$
\left\langle M(f)^{*} \Phi, B \Psi_{0}\right\rangle=\left\langle\Phi, B M(f) \Psi_{0}\right\rangle=\lambda\left\langle\Phi, M(f) \Psi_{0}\right\rangle,
$$

где $\Phi \in D_{0}$, и снова учитывая плотность $D_{0}$ в $\mathcal{H}$, мы получаем $B=\lambda I$, что и требовалось показать.

Аксиому локальной коммутативности заменяем условием асимптотической коммутативности, которое означает, что любые две полевые компоненты либо асимптотически коммутируют, либо асимптотически антикоммутируют. Конечно, это условие слабее локальной коммутативности в том смысле, что оно заведомо выполняется для ограничений локальных полей на подпространство пробных функций, принадлежащих $S_{\alpha}^{0}\left(\mathbb{R}^{4}\right)$. Как обычно, из соображений лоренц-инвариантности считаем, что тип перестановочного соотношения зависит лишь от типа поля, но не от лореншевых индексов, поэтому в дальнейшем эти индексы, как правило, не выписываем.

Рассмотрим обобшенную функцию, определяемую вакуумным средним

$$
\left\langle\Psi_{0}, \phi_{\iota_{1}}\left(x_{1}\right) \ldots \phi_{\iota_{k-1}}\left(x_{k-1}\right)\left[\phi_{\iota_{k}}\left(x_{k}\right), \phi_{\iota_{k+1}}\left(x_{k+1}\right)\right]_{(+)} \phi_{\iota_{k+2}}\left(x_{k+2}\right) \ldots \phi_{\iota_{n}}\left(x_{n}\right) \Psi_{0}\right\rangle,
$$

где знак - или + соответствует типу перестановочного соотношения между полями $\phi_{\iota_{k}}$ и $\phi_{\iota_{k+1}}$. 
ЛЕмма 3. Из условия асимптотической коммутативности вытекает, что определяемый выражсением (12) аналитический функчионал на $S_{\alpha}^{0}\left(\mathbb{R}^{4 n}\right)$ допускает непрерывное продолэсние на пространство $S_{\alpha}^{0}\left(\mathbb{R}^{4(k-1)} \times U \times \mathbb{R}^{4(n-k-1)}\right)$, где $U$ - любой открытый конус в $\mathbb{R}^{8}$ такой, что $\overline{\mathcal{V}} \backslash\{0\} \subset U$. Следовательно, он сосредоточен на замкнутом конусе $\overline{\mathcal{V}}_{n, k}=\mathbb{R}^{4(k-1)} \times \overline{\mathcal{V}} \times \mathbb{R}^{4(n-k-1)}$.

ДокАЗАТЕЛЬСтво. Рассмотрим сначала простейший нетривиальный случай $n=3$, $k=1$. Тогда мы имеем билинейный раздельно непрерывный функционал на $S_{\alpha}^{0}\left(\mathbb{R}^{8}\right) \times$ $S_{\alpha}^{0}\left(\mathbb{R}^{4}\right)$. Условие асимптотической коммутативности означает, что он остается раздельно непрерывным при наделении $S_{\alpha}^{0}\left(\mathbb{R}^{8}\right)$ топологией, индуцированной из $S_{\alpha}^{0}(U)$. Вовсе не очевидно, что продолжение по непрерывности на последнее пространство по первому аргументу, которое возможно при фиксированном втором, снова дает билинейный раздельно непрерывный функционал. Однако пространство $S_{\alpha}^{0}\left(\mathbb{R}^{4}\right)$ бочечно, поскольку является индуктивным пределом пространств Фреше, что позволяет воспользоваться стандартными фактами о продолжении билинейных отображений. Согласно теореме III.5.2 книги [25] рассматриваемьй функционал $\mathfrak{B}$-гипонепрерывен, где $\mathfrak{B}$ - семейство всех ограниченных подмножеств пространства $S_{\alpha}^{0}\left(\mathbb{R}^{8}\right)$ в индуцируемой из $S_{\alpha}^{0}(U)$ топологии. Пространство $S_{\alpha}^{0}\left(\mathbb{R}^{8}\right)$ плотно в $S_{\alpha}^{0}(U)$ по теореме 1, и семейство замыканий в $S_{\alpha}^{0}(U)$ указанных ограниченных множеств очевидно покрывает $S_{\alpha}^{0}(U)$. Поэтому применима теорема III.5.4 книги [25], согласно которой рассматриваемое продолжение действительно билинейно и раздельно непрерывно. Значит, оно может быть отождествлено с непрерывным линейным функционалом на $S_{\alpha}^{0}\left(U \times \mathbb{R}^{4}\right)=S_{\alpha}^{0}(U) \widehat{\otimes}_{i} S_{\alpha}^{0}\left(\mathbb{R}^{4}\right)$, что и требовалось показать.

Пусть теперь $n=4, k=1$. В этом случае рассмотрим выражение (12) как билинейный функционал на $S_{\alpha}^{0}\left(\mathbb{R}^{12}\right) \times S_{\alpha}^{0}\left(\mathbb{R}^{4}\right)$. Прежние соображения показывают, что он раздельно непрерывен при наделении $S_{\alpha}^{0}\left(\mathbb{R}^{12}\right)$ топологией, индуцированной из $S_{\alpha}^{0}(U \times$ $\left.\mathbb{R}^{4}\right)$. Снова повторяя эту аргументацию и используя на сей раз плотность $S_{\alpha}^{0}\left(\mathbb{R}^{12}\right)$ в $S_{\alpha}^{0}\left(U \times \mathbb{R}^{4}\right)$, убеждаемся в существовании единственного непрерывного продолжения на пространство $S_{\alpha}^{0}\left(U \times \mathbb{R}^{8}\right)$. В обшем случае справедливость леммы устанавливается индукцией по $n$.

СлЕДСТВИЕ. Если условие асимптотической коммутативности соблюдается на области $D_{0}$, то оно соблюдается и на бо́льшей области $D_{1}$, образованной конечным линейными комбинациями вакуумного состояния и векторов вида (10).

Как одно из применений теоремы 6 отметим следующий простой факт. При соблюдении условия асимптотической коммутативности замыкание подпространства $L \subset \mathcal{H}$, образованного векторами вида $(10)$ с фиксированными индексами $\iota_{1} \ell_{1}, \ldots, \iota_{n} \ell_{n}$, совпадает с замыканием подпространства, порождаемого при любом другом порядке участвующих полевых операторов. Действительно, обозначим вектор (10) через $\Psi(f)$. Пусть $\pi$-перестановка индексов $1, \ldots, n$, а $\Psi_{\pi}(f)$ - вектор, соответствующий новому порядку операторов. Ортогональность вектора $\Phi$ подпространству $L$ означает, что функционал $\langle\Phi, \Psi(f)\rangle$ тождественно равен нулю. Но тогда функционал $\left\langle\Phi, \Psi_{\pi}(f)\right\rangle$ сосредоточен на 
конусе, отличном от $\mathbb{R}^{4 n}$. Значит, он тоже равен нулю, ибо носитель его фурье-образа лежит в остром конусе в силу спектрального условия.

\section{5. ЛОРЕНЦ-ИНВАРИАНТНАЯ РЕГУЛЯРИЗАЦИЯ}

Пусть $u$ - лоренц-ковариантное ультрараспределение, определенное на пространстве пробных функций $S_{0}^{\alpha}\left(\mathbb{R}^{4}\right), \alpha>1$, и принимаюшее значения в конечномерном векторном пространстве $\mathcal{E}$, в котором задано представление $T$ группы $S L(2, \mathbb{C})$. Для регуляризации поведения $u$ на бесконечности мы будем использовать умножение на инвариантные функции вида

$$
\omega\left(\frac{p}{\mu}\right)=\omega_{0}\left(\frac{p \cdot p}{\mu^{2}}\right)
$$

где $p \cdot p$ - лоренцев квадрат вектора $p$ и $\omega_{0} \in S_{0}^{\alpha^{\prime}}(\mathbb{R}), 1<\alpha^{\prime}<\alpha, \operatorname{supp} \omega_{0} \subset(-1,1)$, и $\omega_{0}(t)=1$ при $|t| \leqslant 1 / 2$.

Поскольку отображение $p \rightarrow p \cdot p$ является аналитическим, функция $\omega$ принадлежит классу Жеврея $C^{L}$ с $L=(n+1)^{\alpha^{\prime}}$ (см. [23, предложение 8.4.1]). Это означает, что на любом компакте $\mathcal{B} \subset \mathbb{R}^{4}$ она удовлетворяет оценке

$$
\left|\partial^{k} \omega(p)\right| \leqslant C_{\mathcal{B}} h_{\mathcal{B}}^{|k|} k^{\alpha^{\prime} k}
$$

Константа $h_{\mathcal{B}}$ растет с увеличением $\mathcal{B}$, и по этой причине, как будет показано ниже, параметр $\alpha^{\prime}$ придется взять отличным от $\alpha$ и как можно более близким к единице. С помошью (14) легко проверяется, что $\omega$ является мультипликатором в $S_{0}^{\alpha}\left(\mathbb{R}^{4}\right)$. Очевидно, что

$$
u_{\text {reg }} \stackrel{\text { def }}{=} u \omega(p / \mu) \rightarrow u
$$

по топологии пространства $S_{0}^{\prime \alpha}\left(\mathbb{R}^{4}, \mathcal{E}\right)$ при $\mu \rightarrow \infty$.

ТеОрема 7. Пусть ультрараспределение $u \in S_{0}^{\prime \alpha}\left(\mathbb{R}^{4}, \mathcal{E}\right)$ лорени-ковариантно и пусть функиия $\omega$ определена посредством (13). Тогда регуляризованный функиионал $u_{\mathrm{reg}}$ (точнее, ограничение $\left.u_{\mathrm{reg}} \mid S_{0}^{\alpha^{\prime}}\right)$ допускает непрерьвное продолжсние на пространство $S_{\alpha-\alpha^{\prime}}^{\alpha^{\prime}}$. В частности, если $\alpha>2$ и $\alpha^{\prime}<\alpha-1$, то преобразование Фурье функционала $u_{\mathrm{reg}}$ строго локализуемо (ибо тогда $S_{\alpha^{\prime}}^{\alpha-\alpha^{\prime}}$ содержит функции с компактным носителем).

ДокАЗАТЕльСтво. Прежде всего отметим, что $S_{0}^{\alpha^{\prime}}$ плотно как в $S_{0}^{\alpha}$, так и в $S_{\alpha-\alpha^{\prime}}^{\alpha^{\prime}}$. Поэтому продолжение, о котором идет речь, единственно. Сначала рассмотрим простейший случай, когда $u$ лоренш-инвариантно. Не ограничивая обшности, можно положить $\mu=1$. Как показано в [26], для выяснения возможности интересуюшего нас продолжения достаточно оценить асимптотическое поведение на бесконечности сглаженного функционала, т.е. свертки $\left(u_{\mathrm{reg}} * g\right)(q)=\left(u_{\mathrm{reg}}, g(q-\cdot)\right), g \in S_{0}^{\alpha^{\prime}}$. Для упрошения выкладок предположим, что $\operatorname{supp} g$ находится в шаре $|p|<1$, считаем, что 
$q^{2}=q^{3}=0$, и воспользуемся конусными переменными $q^{ \pm}=\left(q^{0} \pm q^{1}\right) / \sqrt{2}$. Пусть, кроме того, $\left|q^{-}\right| \leqslant q^{+}, q^{+}>1$. Обозначим через $\Lambda$ преобразование Лоренца в плоскости $\left(p^{0}, p^{1}\right): p^{+} \rightarrow p^{+} / q^{+}, p^{-} \rightarrow q^{+} p^{-}$. Ввиду лоренш-инвариантности $u$ и $\omega$ имеем

$$
\left(u_{\mathrm{reg}} * g\right)(q)=\left(u, g_{q}\right), \quad \text { где } g_{q}(p) \stackrel{\text { def }}{=} \omega(p) g\left(q-\Lambda^{-1} p\right) .
$$

Применим оценку для значения функционала $u \in S_{0}^{\prime \alpha}$ на пробных функциях с носителем в шаре $|p| \leqslant B$, которая имеет вид

$$
\left|\left(u, g_{q}\right)\right| \leqslant\|u\|_{\alpha, A, B}\left\|g_{q}\right\|_{\alpha, A, B}
$$

где по определению топологии $S_{0}^{\alpha}$

$$
\|g\|_{\alpha, A, B}=\sup _{|p| \leqslant B} \sup _{k \in \mathbb{Z}_{+}^{n}} \frac{\left|\partial^{k} g(p)\right|}{A^{|k|} k^{\alpha k}} .
$$

Точки supp $g_{q}$ по построению удовлетворяют неравенствам $|p \cdot p|<1,\left(p^{2}\right)^{2}+\left(p^{3}\right)^{2}<1$, и поэтому $\left|p^{+} p^{-}\right|<1$. Кроме того, $\left|q^{+}-q^{+} p^{+}\right|<1$ и, как следствие, $\left|p^{-}\right|<1 /(1-$ $\left.1 / q^{+}\right)$. Значит, $\operatorname{supp} g_{q}$ содержится в шаре радиуса 2, если $q^{+}$достаточно велико. При оценке производных учитываем, что для функции $g$ конечна норма $\|\cdot\|_{\alpha^{\prime}, a, 1}$ с некоторым $a$ и преобразование $\Lambda^{-1}$ производит сжатие графика функции в $q^{+}$раз по переменной $p^{+}$. Поскольку переход к конусным переменным, как и любое линейное преобразование координат, является автоморфизмом пространств Гельфанда-Шилова, мы находим

$$
\left|\partial^{k} g\left(q-\Lambda^{-1} p\right)\right| \leqslant \sup _{p \in \mathbb{R}^{4}}\left|\partial^{k} g\left(\Lambda^{-1} p\right)\right| \leqslant\|g\|_{\alpha^{\prime}, a, 1}\left(a^{\prime} q^{+}\right)^{|k|} k^{\alpha^{\prime} k}
$$

где $a^{\prime}$ отличается от $a$ на некоторый числовой множитель. (Нетрудно проверить, что $a^{\prime} \leqslant 2^{2 \alpha^{\prime}+1} a$, но это уточнение несушественно для дальнейшего.) Неравенства (14) и (18) вместе с формулой Лейбница дают

$$
\left|\partial^{k} g_{q}(p)\right| \leqslant C\|g\|_{\alpha^{\prime}, a, 1}\left(a^{\prime} q^{+}+h\right)^{|k|} k^{\alpha^{\prime} k},
$$

где $h$ соответствует компакту $|p| \leqslant 2$. Следовательно,

$$
\begin{aligned}
\left|\left(u_{\mathrm{reg}} * g\right)(q)\right| & \leqslant C\|u\|_{\alpha, A, 2}\|g\|_{\alpha^{\prime}, a, 1} \sup _{k} \frac{\left(a^{\prime} q^{+}+h\right)^{|k|}}{A^{|k|} k^{\left(\alpha-\alpha^{\prime}\right) k}} \leqslant \\
& \leqslant C^{\prime}\|g\|_{\alpha^{\prime}, a, 1} \exp \left\{\left(\frac{a^{\prime \prime}|q|}{A}\right)^{\frac{1}{\alpha-\alpha^{\prime}}}\right\} .
\end{aligned}
$$

В других квадрантах плоскости $q^{2}=q^{3}=0$ асимптотическое поведение свертки оценивается аналогично с использованием в качестве параметра буста $\left|q^{+}\right|$или $1 /\left|q^{-}\right|$. При $q$, пробегаюшем ограниченное множество, использование буста излишне и свертка, очевидно, мажорируется константой $C^{\prime \prime}\|g\|_{\alpha^{\prime}, a, 1}$. Далее, любой вектор $q \in \mathbb{R}^{4}$ переводится в некоторую точку $\tilde{q}$ этой плоскости подходящим пространственным поворотом $R$ и 
$\left(u_{\mathrm{reg}} * g\right)(q)=\left(u_{\mathrm{reg}} * g_{R}\right)(\tilde{q})$, где $g_{R}(\cdot)=g\left(R^{-1}(\cdot)\right)$. Соответствие $g \rightarrow g_{R}$ является непрерывным отображением из $S_{0,1}^{\alpha, a}$ в $S_{0,1}^{\alpha, \tilde{a}}$ (где, как легко видеть, $\tilde{a} \leqslant 3 a$ ). Значит, неравенство (19) соблюдается во всем пространстве $\mathbb{R}^{4}$ с другими, быть может, постоянными вместо $C^{\prime}, a^{\prime \prime}$. Поскольку $A$ можно взять сколь угодно большим, оценка (19) означает, что рост $u_{\text {reg }}$ не хуже экспоненциального с порядком $1 /\left(\alpha-\alpha^{\prime}\right)$ и минимальным типом, и согласно предложению 1 работы [26] это позволяет продолжить $u_{\text {reg }}$ на пространство $S_{\alpha-\alpha^{\prime}}^{\alpha^{\prime}}$. Продолжение можно задать формулой

$$
\left(\tilde{u}_{\mathrm{reg}}, g\right)=\int\left(u_{\mathrm{reg}}, \chi_{0}(q-\cdot) g(\cdot)\right) d q, \quad g \in S_{\alpha-\alpha^{\prime}}^{\alpha^{\prime}}
$$

где $\chi_{0}$ - любой элемент $S_{0}^{\alpha^{\prime}}\left(\mathbb{R}^{4}\right)$ с носителем в единичном шаре и свойством

$$
\int \chi_{0}(p) d p=1
$$

Из (19) следует сходимость интеграла в (20) (подробнее см. [26]). В общем случае лоренш-ковариантной обобшенной функции $u_{\ell}$ единственное усложнение связано с зависимостью от $q$ матричных элементов представления $T_{\ell \ell^{\prime}}\left(\Lambda^{-1}\right)$, по которому она преобразуется, в аналоге формулы (15). Однако эта зависимость полиномиально ограничена и не сказывается на экспоненциальных оценках. Доказательство теоремы 7 закончено.

Для случая нескольких переменных $\left(p_{1}, \ldots, p_{n}\right) \in \mathbb{R}^{4 n}$ нам понадобится следуюший результат.

ТЕОРема 8. Пусть и - лорени-ковариантное ультрараспределение со значениями в $\mathcal{E}$, определенное на пространстве $S_{0}^{\alpha}\left(\mathbb{R}^{4 n}\right) . \quad$ Если $\operatorname{supp} u \subset \overline{\mathbb{V}}_{+} \times \cdots$ $\cdots \times \overline{\mathbb{V}}_{+}=\overline{\mathbb{V}}_{+}^{n}$ и регуляризачия производится мультипликатором вида $\omega(p)=$ $\omega_{0}(P \cdot P)$, где $P=\sum_{i=1}^{n} p_{i} u \omega_{0} \in S_{0}^{\alpha^{\prime}}(\mathbb{R}), 1<\alpha^{\prime}<\alpha$, mо для $u_{\mathrm{reg}}=u \omega(p / \mu)$ сохраняют силу утверждения теоремы 7.

ДоказАтельство. Снова рассмотрим свертку $\left(u_{\mathrm{reg}} * g\right)(q), g \in S_{0}^{\alpha^{\prime}}$, считая на этот раз, что supp $g$ находится во множестве $\left\{p \in \mathbb{R}^{4 n}:|p|<1 / n\right\}$. Обозначим $Q=\sum q_{i}$, положим $Q^{2}=Q^{3}=0$ и считаем, что $\left|Q^{-}\right|<Q^{+}, Q^{+}>1$. Воспользуемся преобразованием $\Lambda: p_{i}^{+} \rightarrow p_{i}^{+} / Q^{+}, p_{i}^{-} \rightarrow Q^{+} p_{i}^{-}, i=1, \ldots, n$. В тех точках, где функция $g_{q}=\omega(p) g\left(q-\Lambda^{-1} p\right)$ отлична от нуля, соблюдаются неравенства $|P \cdot P|<1$, $\left(P^{2}\right)^{2}+\left(P^{3}\right)^{2}<1,\left|Q^{+}-Q^{+} P^{+}\right|<1$. Значит, прежнее рассуждение показьвает, что $\operatorname{supp} g_{q}$ содержится во множестве $|P|<2$, если $Q^{+}$достаточно велико. Зададим окрестность $\mathcal{U}$ носителя $u$ в виде объединения окрестности начала с произведением $U^{n}$, где $U-$ открытый, выпуклый, острый конус в $\mathbb{R}^{4}$, содержаший $\overline{\mathbb{V}}_{+} \backslash\{0\}$. Для точек $U^{n}$ соблюдается неравенство $|p|<\theta|P|$ с некоторой константой $\theta$. Действительно, в противном случае найдется последовательность точек $p_{(\nu)} \in U^{n}$ такая, что $\left|p_{(\nu)}\right|=1$ и $\left|P_{(\nu)}\right|<1 / \nu$. Из нее можно выбрать сходяшуюся подпоследовательность, предел которой $\bar{p}$ есть ненулевой вектор в $\bar{U}^{n}$ такой, что $|\bar{P}|=0$, но это противоречит тому, что конус $U$ является острым. Таким образом, множество supp $g_{q} \cap \mathcal{U}$ лежит в шаре радиуса $2 \theta$. Пусть 
$\chi$ - мультипликатор в $S_{0}^{\alpha^{\prime}}$, равный единице в окрестности $\operatorname{supp} u$ и нулю вне $\mathcal{U}$. Тогда $\left(u, g_{q}\right)=\left(u, \chi g_{q}\right)$. Остается оценить норму $\left\|\chi g_{q}\right\|_{\alpha, A, 2 \theta}$. Это сводится к очевидным изменениям в аргументации, применявшейся при выводе неравенства (19), и приводит к такому же результату, что завершает доказательство.

\section{6. РОЛЬ ТОЧЕК ЙОСТА В НЕЛОКАЛЬНОЙ ТЕОРИИ ПОЛЯ}

Напомним, что в локальной теории поля [2-4] точками Йоста называют вешественные точки расширенной области аналитичности вайтмановских функций $\mathcal{W}\left(x_{1}, \ldots, x_{n}\right)$. Это расширение получается по теореме Баргмана-Холла-Вайтмана применением всевозможных комплексных преобразований Лоренца к примитивной области аналитичности, определяемой спектральным условием. В разностных координатах $\xi_{k}=x_{k}-$ $x_{k+1}(k=1, \ldots, n-1)$, от которых фактически зависят вайтмановские функции в силу трансляционной инвариантности, множество точек Йоста записывается следующим образом:

$$
\mathbb{J}_{n-1}=\left\{\xi \in \mathbb{R}^{4(n-1)}:\left(\sum \lambda_{k} \xi_{k}\right)^{2}<0 \quad \forall \lambda_{k} \geqslant 0, \quad \sum \lambda_{k} \neq 0\right\} .
$$

Прообраз этого открытого конуса в $\mathbb{R}^{4 n}$ мы будем обозначать через $\mathcal{J}_{n}$. Если вакуумные средние растут в импульсном пространстве быстрее линейной экспоненты, то область аналитичности в координатном пространстве пуста, поскольку при таком росте преобразование Лапласа не сушествует. Однако, как впервые заметил Люкке [7], точки Йоста продолжают играть важную роль и в этом, сушественно нелокализуемом случае. В данном разделе будет доказано, что при любом высокоэнергетическом поведении полей дополнение к конусу Йоста служит несущим конусом для некоторых комбинаций вакуумных средних, возникаюших при выводе связи спина со статистикой и PCT-симметрии.

Сначала изложим обоснование возможности перехода к разностным переменным, которое в данном случае требует чуть более сложной аргументации, чем в обычной теории умеренного роста [2-4].

ЛЕмма 4. Для всякого трансляционно-инвариантного функционала $\mathcal{W} \in$ $S_{\alpha}^{\prime 0}\left(\mathbb{R}^{4 n}\right)$ существует функционал $W \in S_{\alpha}^{\prime 0}\left(\mathbb{R}^{4(n-1)}\right)$ такой, что

$$
(\mathcal{W}, f)=\left(W, \int f_{t}(\xi) d \xi_{n}\right), \quad \text { əде } \quad f_{t}(\xi)=f\left(\xi_{1}+\cdots+\xi_{n}, \xi_{2}+\cdots+\xi_{n}, \ldots, \xi_{n}\right) .
$$

Принадлежность $W \in S_{\alpha}^{\prime 0}(U)$, где $U$ - открытый конус в $\mathbb{R}^{4(n-1)}$, равносильна принадлежности $\mathcal{W} \in S_{\alpha}^{\prime 0}(\mathcal{U})$, где $\mathcal{U}=\left\{x \in \mathbb{R}^{4 n}:\left(x_{1}-x_{2}, \ldots, x_{n-1}-x_{n}\right) \in U\right\}$.

ДокаЗАТЕЛЬСТво. Линейное преобразование

$$
t: \quad\left(x_{1}, \ldots, x_{n}\right) \rightarrow\left(\xi_{1}=x_{1}-x_{2}, \ldots, \xi_{n-1}=x_{n-1}-x_{n}, \xi_{n}=x_{n}\right),
$$


при котором пробная функция $f(\xi)$ переходит в $f_{t}(\xi)=f\left(t^{-1} \xi\right)$, задает автоморфизм пространства $S_{\alpha}^{0}\left(\mathbb{R}^{4 n}\right)$, а интегрированием по $\xi_{n}$ это пространство непрерывно отображается на $S_{\alpha}^{0}\left(\mathbb{R}^{4(n-1)}\right)$. Следовательно, сопоставляя каждому $W \in S_{\alpha}^{\prime 0}\left(\mathbb{R}^{4(n-1)}\right)$ функционал $\mathcal{W}$ по формуле $(22)$, мы получаем инъективное отображение $S_{\alpha}^{\prime 0}\left(\mathbb{R}^{4(n-1)}\right) \rightarrow$ $S_{\alpha}^{\prime 0}\left(\mathbb{R}^{4 n}\right)$, которое очевидно непрерывно при наделении сопряженных пространств слабыми топологиями. Лемма утверждает, что любой трансляционно-инвариантный функционал $\mathcal{W}$ принадлежит образу этого отображения. И в самом деле, его регуляризация посредством свертки с $\delta$-образной последовательностью пробных функций дает последовательность $\mathcal{W}_{\nu}$, для которой заведомо справедливо представление $(22)$ с гладкими функциями $W_{\nu}$. Последовательность $W_{\nu}$ слабо фундаментальна и, поскольку пространство $S_{\alpha}^{\prime 0}$ монтелевское ${ }^{5)}$, сходится в нем к некоторому $W$, образом которого является $\mathcal{W}$.

Столь же очевидна справедливость второго утверждения леммы, поскольку при преобразовании (23) подпространство функционалов, инвариантных относительно одновременного сдвига всех аргументов, переходит в подпространство функционалов, инвариантных относительно трансляций по последнему аргументу, а индикаторную функцию, задаюшую $S_{\alpha}^{\prime 0}(\mathcal{U})$, можно взять мультипликативной, в виде произведения функции, зависящей от $\xi_{n}$, и функции остальных $\xi_{k}$ (см. $\left.[12, \S 3]\right)$, что завершает доказательство.

Из леммы 4 вытекает, конечно, что если $K$ - несущий конус функционала $W$, то его прообраз $\mathcal{K}$ в $\mathbb{R}^{4 n}$ является несущим конусом $\mathcal{W}$. Действительно, если открытый конус $U$ содержит конус $K \backslash\{0\}$ и стягивается к нему, то $\mathcal{U}$ содержится в любой наперед заданной конусной окрестности $\mathcal{K}$.

ТЕОРема 9. Пусть поле ф определено на пространстве $S_{\alpha}^{0}\left(\mathbb{R}^{4}\right), \alpha>2$, и преобразуется по неприводимому представлению группы $S L(2, \mathbb{C})$. Пусть $\mathcal{W}\left(x_{1}, x_{2}\right)-$ вайтмановская обобщенная функиия, определяемая вакуумным средним $\left\langle\Psi_{0}\right.$, $\left.\phi\left(x_{1}\right) \phi^{*}\left(x_{2}\right) \Psi_{0}\right\rangle$. Если $\phi$ имеет иельй спин, то вследствие пуанкаре-ковариантности и спектрального условия конус $\overline{\mathcal{V}}=\complement \mathcal{J}_{2}$ является несущим для разности

$$
\mathcal{W}\left(x_{1}, x_{2}\right)-\mathcal{W}\left(x_{2}, x_{1}\right)
$$

В случае получелого спина он является несущим для суммы

$$
\mathcal{W}\left(x_{1}, x_{2}\right)+\mathcal{W}\left(x_{2}, x_{1}\right)
$$

ДокАЗАТЕЛЬСТво. Лемма 4 сводит задачу к выводу соответствующих свойств для функционала $W \in S_{\alpha}^{\prime 0}\left(\mathbb{R}^{4}\right)$. Воспользуемся рассмотренной выше ультрафиолетовой регуляризацией и обозначим $\check{W}_{\mu}(p)=\check{W}(p) \omega(p / \mu)$, где $\omega$ выбрано указанным в разделе 5 способом. В силу спектрального условия supp $\check{W}_{\mu}$ лежит в конусе $\overline{\mathbb{V}}_{+}$, а согласно теореме 7 функционал $\check{W}_{\mu}$ определен на пространстве $S_{\alpha-\alpha^{\prime}}^{\alpha^{\prime}}$, где $\alpha-\alpha^{\prime}>1$, т.е. рост $\breve{W}_{\mu}$ на бесконечности не хуже экспоненциального с порядком $<1$. Поэтому $\check{W}_{\mu}$ имеет (обратное) преобразование Лапласа $\mathbf{W}_{\mu}(\zeta)$, которое голоморфно в обычной трубчатой

\footnotetext{
5) В [21] вместо этого, принятого сейчас термина используется термин "совершенное".
} 
области $\mathbb{T}_{+}=\mathbb{R}^{4}-i \mathbb{V}_{+}$и граничным значением которого служит $W_{\mu} \in S_{\alpha^{\prime}}^{\prime \alpha-\alpha^{\prime}}$ (см., например, теорему 4 работы [26], где изложены детали распространения теоремы Пэли-Винера-Шварца на обобщенные функции такого класса). Поскольку регуляризация сохраняет лоренц-ковариантность, к аналитической функции $\mathbf{W}_{\mu}(\zeta)$ применима теорема Баргмана-Холла-Вайтмана [2-4], согласно которой такая функция допускает аналитическое продолжение в расширенную область $\mathbb{T}_{+}^{\text {ext }}$, и это продолжение ковариантно относительно комплексной группы Лоренца $L_{+}(\mathbb{C})$. Для рассматриваемой комбинации полей $\phi \phi^{*}$ закон преобразования аналитической функции Вайтмана относительно полного отражения $P T \in L_{+}(\mathbb{C})$ имеет вид

$$
\mathbf{W}_{\mu}(\zeta)= \pm \mathbf{W}_{\mu}(-\zeta)
$$

здесь и далее верхний знак отвечает случаю целого спина, а нижний - случаю полуцелого. Это свойство симметрии является ключевым, как и в классическом выводе теоремы о связи спина со статистикой [2-4]. Поскольку $\mathbb{T}_{+}^{\text {ext }}$ содержит все пространственноподобные точки, из (26) вытекает, что обобщенная функция $F_{\mu} \stackrel{\text { def }}{=} W_{\mu}(\xi) \mp W_{\mu}(-\xi)$ сосредоточена в замкнутом световом конусе $\overline{\mathbb{V}}$ и, следовательно, допускает непрерывное продолжение на пространство $S_{\alpha^{\prime}}^{0}(\mathbb{V})$. Действительно, это продолжение можно задать формулой $\left(\widetilde{F}_{\mu}, f\right)=\left(F_{\mu}, \chi f\right)$, где $\chi$ - мультипликатор в пространстве $S_{\alpha^{\prime}}^{\alpha-\alpha^{\prime}}$, тождественно равный единице в $\epsilon$-окрестности $\overline{\mathbb{V}}$ и обращающийся в нуль вне $2 \epsilon$-окрестности. Такой мультипликатор удовлетворяет оценке

$$
\left|\partial^{q} \chi(x)\right| \leqslant C h^{|q|} q^{\left(\alpha-\alpha^{\prime}\right) q}
$$

а для функции $f \in S_{\alpha^{\prime}}^{0}(\mathbb{V})$ всюду на его носителе в силу формулы Тейлора соблюдаются неравенства

$$
\left|\partial^{q} f(x)\right| \leqslant C_{\epsilon}\|f\|_{a, b} b^{|q|} \exp \left\{-\left|\frac{x}{a}\right|^{\frac{1}{\alpha^{\prime}}}\right\} .
$$

Поэтому умножение на $\chi$ непрерьвно отображает $S_{\alpha^{\prime}}^{0}(\mathbb{V})$ в $S_{\alpha^{\prime}}^{\alpha-\alpha^{\prime}}$. Важно, что продолжения $\widetilde{F}_{\mu}$ согласованы друг с другом, а именно,

$$
\widetilde{F}_{\mu}\left|S_{\alpha^{\prime}, a}^{0, b}(\mathbb{V})=\widetilde{F}_{\mu^{\prime}}\right| S_{\alpha^{\prime}, a}^{0, b}(\mathbb{V})
$$

если $\mu, \mu^{\prime}$ достаточно велики по сравнению с $b$. В самом деле, $\left(W_{\mu}, f\right)=(W, f)$ для $f \in S_{\alpha^{\prime}, a^{\prime}}^{0, \mu / 4}$ с любым $a^{\prime}$, поскольку тогда

$$
|f(z)| \leqslant C \exp \left\{-\left|\frac{x}{a^{\prime}}\right|^{\frac{1}{\alpha^{\prime}}}+\left(\frac{\mu}{4}\right) \sum\left|y_{i}\right|\right\}
$$

а из этой оценки следует (снова в силу теоремы типа Пэли-Винера-Шварца, на этот раз в ее простейшем варианте, для функций из $\mathcal{D}$, см., например, теорему 7.3.1 книги [23]), 
что supp $\check{f}$ содержится в шаре $|p| \leqslant \mu / 2$, где $\omega(p / \mu)=1$ по построению раздела 5 . Согласно подробной формулировке теоремы 1 о плотности, данной в работе [12], существует константа $c$ такая, что при $b^{\prime}>c b, a^{\prime}>c a$ пространство $S_{\alpha^{\prime}, a^{\prime}}^{0, b^{\prime}}$ плотно в $S_{\alpha^{\prime}, a}^{0, b}(\mathbb{V})$ по топологии $S_{\alpha^{\prime}, a^{\prime}}^{0, b^{\prime}}(\mathbb{V})$. Значит, равенство (28) заведомо соблюдается при $\mu, \mu^{\prime}>4 c b$. Таким образом, и нерегуляризованный функционал $W(\xi) \mp W(-\xi)$ допускает непрерывное продолжение на $S_{\alpha^{\prime}}^{0}(\mathbb{V})$. Применение теоремы 5 и леммы 4 завершает доказательство.

Теорема 9 является частным случаем следуюшего более общего утверждения.

ТеОрема 10. Пусть поля $\left\{\phi_{l}\right\}$ определены на пространстве пробных функиий $S_{\alpha}^{0}\left(\mathbb{R}^{4}\right), \quad \alpha>2$, и преобразуются по неприводимыц представлениям $\left(j_{\iota}, k_{\iota}\right)$ группы $S L(2, \mathbb{C})$. Пусть $\mathcal{W}_{\iota_{1} \ldots \iota_{n}}$ - вайтмановская функиия, определяемая $n$-точечным

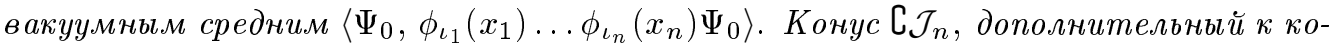
нусу Йоста, является несущим для обобщенной функиии

$$
\mathcal{W}_{\iota_{1} \ldots \iota_{n}}\left(x_{1}, \ldots, x_{n}\right)-(-1)^{2 J} \mathcal{W}_{\iota_{1} \ldots \iota_{n}}\left(-x_{1}, \ldots,-x_{n}\right),
$$

где $J=j_{\iota_{1}}+\cdots+j_{\iota_{n}}$.

Доказательство полностью аналогично только что изложенному с той разницей, что теперь надо воспользоваться режушей функцией вида $\omega(P / \mu)$, где $P=p_{1}+\cdots+p_{n}$, привлечь теорему 8 вместо теоремы 7 и применить известный общий закон преобразования $n$-точечной аналитической функции Вайтмана неприводимых полей относительно отражения.

\section{7. ОБОБШЕНИЕ ТЕОРЕМЫ О СВЯЗИ СПИНА СО СТАТИСТИКОЙ}

Начнем с вывода аналога леммы Дел'Антонио, согласно которой любая пара ненулевых локальных полей $\phi, \psi$ имеет тот же тип перестановочных соотношений, что и пара $\phi, \psi^{*}$.

Теорема 11. Пусть поля $\phi, \psi$, как и әрмитово сопряженные $к$ ним, определены на пространстве пробных функций $S_{\alpha}^{0}\left(\mathbb{R}^{4}\right), \quad \alpha>1$. Если ф имеет разные асимптотические перестановочнъе соотношения с $\psi$ и $\psi^{*}$, то либо $\phi(x) \Psi_{0}=0$, либо $\psi(x) \Psi_{0}=0$.

ДокАЗАТЕЛЬСТво. Считаем для определенности, что $\phi$ асимптотически коммутирует с $\psi$ и асимптотически антикоммутирует с $\psi^{*}$ при пространственноподобном разделении аргументов. Рассмотрим сумму вакуумных средних

$$
\begin{aligned}
\left\langle\Psi_{0}, \phi^{*}\left(x_{1}\right) \phi\left(x_{2}\right) \psi^{*}\left(y_{1}\right) \psi\left(y_{2}\right) \Psi_{0}\right\rangle+\left\langle\Psi_{0}, \phi^{*}\left(x_{1}\right) \psi^{*}\left(y_{1}\right) \psi\left(y_{2}\right) \phi\left(x_{2}\right) \Psi_{0}\right\rangle= & \\
= & \left\langle\Psi_{0}, \phi^{*}\left(x_{1}\right)\left[\phi\left(x_{2}\right), \psi^{*}\left(y_{1}\right)\right]_{+} \psi\left(y_{2}\right) \Psi_{0}\right\rangle+ \\
& +\left\langle\Psi_{0}, \phi^{*}\left(x_{1}\right) \psi^{*}\left(y_{1}\right)\left[\psi\left(y_{2}\right), \phi\left(x_{2}\right)\right]_{-} \Psi_{0}\right\rangle .
\end{aligned}
$$

В силу леммы 3 эта обобшенная функция сосредоточена на объединении конусов $\{(x, y)$ : $\left.\left(x_{2}-y_{1}\right)^{2} \geqslant 0\right\}$ и $\left\{(x, y):\left(x_{2}-y_{2}\right)^{2} \geqslant 0\right\}$. Усредним ее с пробной функцией вида

$$
\bar{f}\left(x_{1}\right) f\left(x_{2}\right) \bar{g}\left(y_{1}-\lambda r\right) g\left(y_{2}-\lambda r\right),
$$


где $r$ - фиксированный пространственноподобный вектор и $\lambda>0$. Результат усреднения есть не что иное, как свертка, рассматриваемая на луче $x_{1}=x_{2}=0, y_{1}=y_{2}=\lambda r$ и согласно лемме 1 он убывает при $\lambda \rightarrow \infty$, поскольку этот луч не принадлежит несушему конусу. С другой стороны, как и в оригинальном рассуждении Дел'Антонио, результат усреднения левой части соотношения (30) можно представить следуюшим образом:

$$
\left\langle\Psi_{0}, \phi(f)^{*} \phi(f) T(\lambda r) \psi(g)^{*} \psi(g) \Psi_{0}\right\rangle+\left\|\psi(g) T^{-1}(\lambda r) \phi(f) \Psi_{0}\right\|^{2},
$$

где $T(\lambda r)$ - оператор трансляции. При $\lambda \rightarrow \infty$ первое слагаемое стремится к

$$
\left\|\phi(f) \Psi_{0}\right\|^{2}\left\|\psi(g) \Psi_{0}\right\|^{2}
$$

ввиду кластерного свойства, которое, как известно, можно вывести из аксиом Вайтмана, никоим образом не используя локальность $[2,3]$. Таким образом, если $\psi(g) \Psi_{0} \neq 0$ хотя бы для одной пробной функции $g$, то $\phi(f) \Psi_{0}=0$ при всех $f \in S_{\alpha}^{0}\left(\mathbb{R}^{4}\right)$, что завершает доказательство.

ТЕОРемА 12. Пусть поле ф определено на пространстве пробных функиий $S_{\alpha}^{0}\left(\mathbb{R}^{4}\right)$ с индексом $\alpha>2$ и преобразуется по неприводимому представлению груп$n$ b $S L(2, \mathbb{C})$. Аномальное асимптотическое перестановочное соотношение мех$\partial y \phi$ и әрмитово сопряженным полем $\phi^{*}$ (антикоммутативность в случае иелого спина и коммутативность в случае полуцелого) приводит к равенству $\phi(f) \Psi_{0}=\phi^{*}(f) \Psi_{0}=0$ для всех $f \in S_{\alpha}^{0}\left(\mathbb{R}^{4}\right)$

ДоказАтельство. Пусть $\phi-$ поле целого спина. Аномальное перестановочное соотношение означало бы, в частности, что сумма

$$
\left\langle\Psi_{0}, \phi^{*}\left(x_{1}\right) \phi\left(x_{2}\right) \Psi_{0}\right\rangle+\left\langle\Psi_{0}, \phi\left(x_{2}\right) \phi^{*}\left(x_{1}\right) \Psi_{0}\right\rangle
$$

сосредоточена на конусе $\overline{\mathcal{V}}$. Из теоремы 9 следует, что тогда этот конус является несущим и для суммы

$$
\left\langle\Psi_{0}, \phi^{*}\left(x_{1}\right) \phi\left(x_{2}\right) \Psi_{0}\right\rangle+\left\langle\Psi_{0}, \phi\left(x_{1}\right) \phi^{*}\left(x_{2}\right) \Psi_{0}\right\rangle .
$$

В импульсном представлении оба входящие в (32) вакуумные средние имеют носитель в остром конусе $\left\{p \in \mathbb{R}^{8}: p_{1}+p_{2}=0, p_{1} \in \overline{\mathbb{V}}_{+}\right\}$. Значит, по теореме 6 эта сумма равна нулю. Взяв ее значение на пробной функции $\bar{f}\left(x_{1}\right) f\left(x_{2}\right)$, получаем

$$
\left\|\phi(f) \Psi_{0}\right\|^{2}+\left\|\phi^{*}(f) \Psi_{0}\right\|^{2}=0 .
$$

В случае полуцелого спина рассуждение такое же, с соответствующим изменением знаков в формулах. Теорема доказана.

СледСТВИЕ. В теории поля с пробными функииями из пространства $S_{\alpha}^{0}\left(\mathbb{R}^{4}\right)$, $\alpha>2$, подчиненной условию асимптотической коммутативности, справедливо равенство

$$
\left\langle\Psi_{0}, \phi^{*}\left(x_{1}\right) \phi\left(x_{2}\right) \Psi_{0}\right\rangle=\left\langle\Psi_{0}, \phi\left(x_{1}\right) \phi^{*}\left(x_{2}\right) \Psi_{0}\right\rangle .
$$


ДокАЗАТЕльСтво. Разность этих вакуумных средних можно записать как

$$
\left\langle\Psi_{0},\left[\phi^{*}\left(x_{1}\right) \phi\left(x_{2}\right)\right]_{\mp} \Psi_{0}\right\rangle \pm\left\langle\Psi_{0}, \phi\left(x_{2}\right) \phi^{*}\left(x_{1}\right) \Psi_{0}\right\rangle-\left\langle\Psi_{0}, \phi\left(x_{1}\right) \phi^{*}\left(x_{2}\right) \Psi_{0}\right\rangle .
$$

В силу теоремы 9 как в случае целого, так и в случае полуцелого спина выражение (34) сосредоточено на конусе $\overline{\mathcal{V}}$, а в силу теоремы 6 это свойство совместно со спектральным условием лишь тогда, когда соблюдается (33).

ТЕОрема 13. В теории поля с пробными функииями из пространства $S_{\alpha}^{0}\left(\mathbb{R}^{4}\right)$, $\alpha>2$, подчиненной условию асимптотической коммутативности, соблюдение

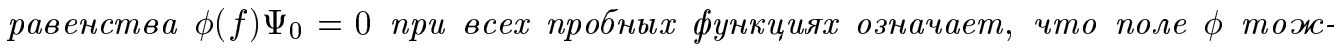
дественно равно нулю.

ДокАЗАТЕЛЬСТво. Из предположения теоремы следует, что равны нулю любые вакуумные средние, содержащие хотя бы один оператор $\phi$. Пусть, например, $\phi$ стоит на предпоследнем месте. Тогда вакуумное среднее

$$
\left\langle\Psi_{0}, \phi_{\iota_{1}}, \ldots, \phi_{\iota_{n-1}} \phi \phi_{\iota_{n}} \Psi_{0}\right\rangle
$$

совпадает с обобщенной функцией

$$
\left\langle\Psi_{0}, \phi_{\iota_{1}}, \ldots, \phi_{\iota_{n-1}}\left[\phi, \phi_{\iota_{n}}\right]_{\mp} \Psi_{0}\right\rangle
$$

сосредоточенной на конусе $\mathbb{R}^{4(n-1)} \times \overline{\mathcal{V}}$, а носитель его преобразования Фурье лежит в остром конусе, определяемом спектральным условием. Значит, оно равно нулю в силу теоремы 6. Далее применяем индукцию. Из аксиомы цикличности вакуума вытекает теперь, что $\langle\Phi, \phi(f) \Psi\rangle=0$ при всех $\Phi \in \mathcal{H}, \Psi \in D_{0}, f \in S_{\alpha}^{0}\left(\mathbb{R}^{4}\right)$. Оператор $\phi(f)$ допускает замыкание, поскольку сопряженный ему оператор плотно определен. Значит, $\phi(f)=0$, что и утверждалось.

Отметим, что это рассуждение сохраняет силу при замене $\phi$ на любой моном $M$ по компонентам полей, поэтому условие $M \Psi_{0}=0$ влечет $M=0$, что, конечно, можно рассматривать и как следствие теоремы 13 и кластерного свойства.

Нам понадобится еше следуюший простой факт, который устанавливается аналогично теореме 11.

ТЕОРема 14. Пусть $\left\{\phi_{\iota}\right\}$ - система полей, определенньх на пространстве $S_{\alpha}^{0}\left(\mathbb{R}^{4}\right), \alpha>1$. Если два монома $M=\phi_{\iota_{1}}\left(x_{1}\right) \ldots \phi_{\iota_{m}}\left(x_{m}\right)$ u $N=\phi_{\iota_{1}^{\prime}}\left(y_{1}\right) \ldots \phi_{\iota_{n}^{\prime}}\left(y_{n}\right)$ по компонентам полей из этого набора асимптотически антикоммутируют при пространственноподобном разделении аргументов $x=\left(x_{1}, \ldots, x_{m}\right)$ u $y=\left(y_{1}, \ldots\right.$ $\left.\ldots, y_{n}\right)$, mо либо $\left\langle\Psi_{0}, M \Psi_{0}\right\rangle \equiv 0, л u б о\left\langle\Psi_{0}, N \Psi_{0}\right\rangle \equiv 0$. 
ДокАЗАТЕЛЬСТво. В данном случае мы имеем обобщенную функцию

$$
\left\langle\Psi_{0}, M\left(x_{1}, \ldots, x_{m}\right) N\left(y_{1}, \ldots, y_{n}\right) \Psi_{0}\right\rangle+\left\langle\Psi_{0}, N\left(y_{1}, \ldots, y_{n}\right) M\left(x_{1}, \ldots, x_{m}\right) \Psi_{0}\right\rangle
$$

с несущим конусом

$$
\bigcup_{\substack{k=1, \ldots, m \\ l=1, \ldots, n}}\left\{(x, y) \in \mathbb{R}^{4(m+n)}:\left(x_{k}-y_{l}\right)^{2} \geqslant 0\right\} .
$$

Усреднение с пробной функцией вида $f\left(x_{1}, \ldots, x_{m}\right) g\left(y_{1}-\lambda r, \ldots, y_{n}-\lambda r\right)$ дает, как и прежде, гладкую функцию параметра $\lambda$, которая быстро убывает при $\lambda \rightarrow \infty$ согласно лемме 1. С другой стороны, эту функцию можно записать как

$$
\left\langle\Psi_{0}, M(f) T(\lambda r) N(g) \Psi_{0}\right\rangle+\left\langle\Psi_{0}, N(g) T^{-1}(\lambda r) M(f) \Psi_{0}\right\rangle,
$$

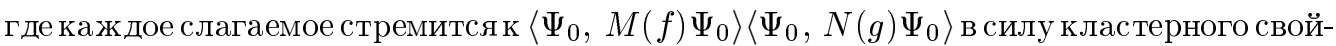
ства. Значит, это произведение вакуумных средних равно нулю при всех $f \in S_{\alpha}^{0}\left(\mathbb{R}^{4 m}\right)$, $g \in S_{\alpha}^{0}\left(\mathbb{R}^{4 n}\right)$. Теорема доказана.

Теперь мы полностью подготовлены к выводу аналога теоремы Араки о приведении перестановочных соотношений к нормальному виду. Мы придерживаемся обычного соглашения [2], что среди рассматриваемых полей нет тождественно равных нулю и что в том случае, когда поле $\phi_{\iota}$ не является эрмитовым, сопряженное поле $\phi_{\iota}^{*}$ входит в рассматриваемый набор с некоторым индексом $\bar{\iota} \neq \iota$.

Теорема 15. В теории вайтмановских полей $\left\{\phi_{\iota}\right\}, \iota=1, \ldots, I$, определенных на пространстве пробных функиий $S_{\alpha}^{0}\left(\mathbb{R}^{4}\right)$ с индексом $\alpha>2$ и подчиненных условию асимптотической коммутативности, существует преобразование Клейна $\phi_{\iota} \Rightarrow \phi_{\iota}^{\prime}$, приводящее перестановочные соотношения $к$ нормальному виду, т.е. при пространственноподобном разделении аргументов поля $\phi_{\iota}^{\prime}$ с челым спином асимптотически коммутируют с любыми полями из нового набора, а преобразованные поля с полуцельм спином асимптотически антикоммутируют между собой. При этом поля $\phi_{\iota}^{\prime}$ удовлетворяют всем остальныцм аксиомам Вайтмана и сохраняется условие әрмитова сопряэсения $\phi_{\llcorner}^{\prime *}=\phi_{\bar{L}}^{\prime}$.

ДоКАЗАТЕЛЬСТво. Теоремы 11-14 сводят доказательство этого утверждения к практически дословному повторению классического вывода [2-4] самой теоремы Араки.

Обозначим через $F_{\iota}$ спинорное число поля $\phi_{\iota}$. Условие асимптотической коммутативности означает, что для любой пары полей $\phi_{\iota}, \phi_{\iota^{\prime}}$ из рассматриваемого набора матричные элементы комбинации

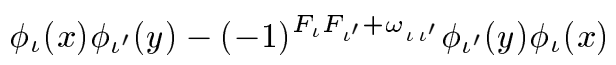

сосредоточены на конусе $\overline{\mathcal{V}}$. Здесь $\omega_{\iota \iota^{\prime}}=0$, если перестановочное соотношение нормально, и $\omega_{\iota \iota^{\prime}}=1$ в противном случае. Матрица $\omega$ симметрична и в силу теорем 11 и 12 имеет свойства

$$
\omega_{\iota \iota^{\prime}}=\omega_{\bar{\iota} \iota^{\prime}}=\omega_{\iota \bar{\iota}^{\prime}}, \quad \omega_{\iota \iota}=0 .
$$


Она определяет знак в перестановочном соотношении для любых двух мономов по полям, причем этот знак зависит лишш от того, какое число полей каждого типа входит в мономы, а точнее, лишь от четности этих чисел. Эту характеристику монома $M$ можно записать в виде строки $\mathfrak{m}=\left(\mathfrak{m}^{1}, \ldots, \mathfrak{m}^{I}\right)$, где $\mathfrak{m}^{\iota}=0$, если поле $\phi_{\iota}$ входит в $M$ четное число раз, и $\mathfrak{m}^{\iota}=1$ в противном случае. Множество $\mathfrak{M}$ таких строк имеет структуру векторного пространства над полем $\mathbb{Z}_{2}$, и отображение $M \rightarrow \mathfrak{m}$ согласовано с этой структурой в том смысле, что

$$
\mathfrak{m}\left(M_{1} M_{2}\right)=\mathfrak{m}\left(M_{1}\right)+\mathfrak{m}\left(M_{2}\right) .
$$

Набор спинорных чисел задает линейную форму $F(\mathfrak{m})=\sum F_{\iota} \mathfrak{m}^{\iota}$, а матрица $\omega$ определяет билинейную форму $\left(\mathfrak{m}_{1}, \mathfrak{m}_{2}\right)=\sum \omega_{\iota} \iota^{\prime} \mathfrak{m}^{\iota} \mathfrak{m}^{\iota^{\prime}}$ на $\mathfrak{M}$. В этих обозначениях знаковый множитель в перестановочном соотношении для мономов $M_{1}, M_{2}$ имеет вид

$$
(-1)^{F\left(\mathfrak{m}_{1}\right) F\left(\mathfrak{m}_{2}\right)+\left(\mathfrak{m}_{1}, \mathfrak{m}_{2}\right)},
$$

а свойства (36) записываются следуюшим образом:

$$
\left(\mathfrak{e}_{\iota}, \mathfrak{m}\right)=\left(\mathfrak{e}_{\bar{\iota}}, \mathfrak{m}\right), \quad(\mathfrak{m}, \mathfrak{m})=0
$$

где $\mathfrak{e}_{\iota}=\mathfrak{m}\left(\phi_{\iota}\right)$. Поскольку в случае поля $\mathbb{Z}_{2}$ равенства $\left(\mathfrak{m}_{1}, \mathfrak{m}_{2}\right)=\left(\mathfrak{m}_{2}, \mathfrak{m}_{1}\right)$ и $\left(\mathfrak{m}_{1}, \mathfrak{m}_{2}\right)+$ $\left(\mathfrak{m}_{2}, \mathfrak{m}_{1}\right)=0$ эквивалентны, вторая из формул $(38)$ означает, что форма $(\cdot, \cdot)$ порождает симплектическую структуру в $\mathfrak{M}$. Обозначим через $\mathfrak{A}$ множество тех векторов в $\mathfrak{M}$, которые отвечают мономам с не равными тождественно нулю вакуумными средними. Из (37) и кластерного свойства следует, что $\mathfrak{A}$ является линейным подпространством. Ограничение на $\mathfrak{A}$ формы $(\cdot, \cdot)$ есть нуль, поскольку такие мономы содержат четное число спинорных полей, и равенство $\left(\mathfrak{m}_{1}, \mathfrak{m}_{2}\right)=1$ означало бы, что $M_{1}$ и $M_{2}$ антикоммутируют при пространственноподобном разделении аргументов, что по теореме 14 приводило бы к противоречию. Любой базис $\left(\mathfrak{a}_{1}, \ldots, \mathfrak{a}_{q}\right)$ в $\mathfrak{A}$ можно пополнить до симплектического базиса $\left(\mathfrak{a}_{1}, \ldots, \mathfrak{a}_{r} ; \mathfrak{b}_{1}, \ldots, \mathfrak{b}_{r} ; \mathfrak{c}_{1}, \ldots, \mathfrak{c}_{s}\right), r \geqslant q, 2 r+s=I$, во всем множестве $\mathfrak{M}$. Этот базис характеризуется тем, что

$$
\left(\mathfrak{a}_{j}, \mathfrak{b}_{j}\right)=1, \quad j=1, \ldots, r
$$

а остальные спаривания дают нуль. В частности,

$$
\left(\mathfrak{a}_{j}, \mathfrak{m}\right)=0 \text { при всех } j=1, \ldots, r, \quad \mathfrak{m} \in \mathfrak{A} .
$$

Каждому $\mathfrak{a}_{j}$ можно сопоставить оператор $\theta_{j}$ в гильбертовом пространстве состояний $\mathcal{H}$, полагая

$$
\theta_{j} \Psi_{0}=\Psi_{0}, \quad \theta_{j} M(f) \Psi_{0}=(-1)^{\left(\mathfrak{a}_{j}, \mathfrak{m}\right)} M(f) \Psi_{0} .
$$

Такое определение корректно, ибо $M_{1}\left(f_{1}\right) \Psi_{0}=M_{2}\left(f_{2}\right) \Psi_{0} \neq 0$ влечет $\left(\mathfrak{a}_{j}, \mathfrak{m}_{1}\right)=\left(\mathfrak{a}_{j}\right.$, $\left.\mathfrak{m}_{2}\right)$. Действительно, тогда $\left\langle\Psi_{0}, M_{1}^{*} M_{2} \Psi_{0}\right\rangle \not \equiv 0$ и применение (37)-(39) дает

$$
\omega\left(\mathfrak{a}_{j}, \mathfrak{m}_{1}\right)+\omega\left(\mathfrak{a}_{j}, \mathfrak{m}_{2}\right)=0 .
$$


Далее, определение согласовано с линейными операциями в $\mathcal{H}$, так как из равенства $M(f) \Psi_{0}=M_{1}\left(f_{1}\right) \Psi_{0}+M_{2}\left(f_{2}\right) \Psi_{0}$, где каждый из векторов отличен от нуля, следует равенство $\left(\mathfrak{a}_{j}, \mathfrak{m}\right)=\left(\mathfrak{a}_{j}, \mathfrak{m}_{1}\right)=\left(\mathfrak{a}_{j}, \mathfrak{m}_{2}\right)$. В самом деле, если, например, $\left(\mathfrak{a}_{j}, \mathfrak{m}_{1}\right)$ отлично от двух других скалярных произведений, то, снова используя (37)-(39), мы получаем противоречие, поскольку хотя бы одно из вакуумных средних $\left\langle\Psi_{0}, M_{1}^{*} M \Psi_{0}\right\rangle$, $\left\langle\Psi_{0}, M_{1}^{*} M_{2} \Psi_{0}\right\rangle$ не равно нулю тождественно. Значит, оператор $\theta_{j}$ можно продолжить по линейности на $D_{0}$. Из (37)-(39) следует, что $\left\langle\theta_{j} \Phi, \theta_{j} \Psi\right\rangle=\langle\Phi, \Psi\rangle$ при всех $\Phi, \Psi \in D_{0}$. Поэтому $\theta_{j}$ однозначно продолжается до унитарного и, очевидно, инволютивного оператора в $\mathcal{H}$. Полагаем

$$
U_{\iota}=\prod_{j=1}^{r} \theta_{j}^{\left(\mathfrak{e}_{\iota}, \mathfrak{b}_{j}\right)}, \quad \iota=1, \ldots, I .
$$

Операторы $U_{\iota}$ взаимно коммутируют и имеют следуюшие перестановочные соотношения с полями:

$$
U_{\iota} \phi_{\iota^{\prime}}=(-1)^{\sigma_{\iota \iota^{\prime}}} \phi_{\iota^{\prime}} U_{\iota}, \quad \text { где } \sigma_{\iota \iota^{\prime}}=\sum_{j=1}^{r}\left(\mathfrak{e}_{\iota}, \mathfrak{b}_{j}\right)\left(\mathfrak{a}_{j}, \mathfrak{e}_{\iota^{\prime}}\right)
$$

Преобразование Клейна определяется формулой

$$
\phi_{\iota} \Rightarrow \phi_{\iota}^{\prime}=i^{\sigma_{\iota \iota}} U_{\iota} \phi_{\iota} .
$$

Из (41) вместе с равенством $\omega_{\iota \iota^{\prime}}=\sigma_{\iota \iota^{\prime}}+\sigma_{\iota^{\prime} \iota}$, которое соблюдается по определению симплектического базиса, следует, что при любых $\Phi, \Psi \in D_{0}$

$$
\begin{aligned}
& \left\langle\Phi,\left[\phi_{\iota}^{\prime}(x) \phi_{\iota^{\prime}}^{\prime}(y)-(-1)^{F_{\iota} F_{\iota^{\prime}}} \phi_{\iota^{\prime}}^{\prime}(y) \phi_{\iota}^{\prime}(x)\right] \Psi\right\rangle=
\end{aligned}
$$

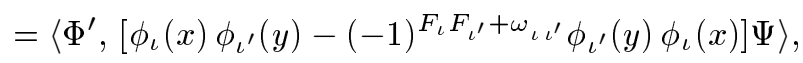

где $\Phi^{\prime}=(-1)^{\sigma \iota^{\prime} \iota}(-i)^{\sigma \iota \iota}+\sigma_{\iota^{\prime} \iota^{\prime}} U_{\iota} U_{\iota^{\prime}} \Phi \in D_{0}$. Таким образом, преобразованные поля удовлетворяют нормальным асимптотическим перестановочным соотношениям. Из (38), (41) видно, что присутствие в формуле (42) множителя $i^{\sigma_{\iota \iota}}$ обеспечивает равенство $\phi_{\iota}^{\prime *}=\phi_{\bar{\iota}}^{\prime}$.

Не составляет труда проверка остальных аксиом Вайтмана для полей $\phi_{\iota}^{\prime}$, что завершает доказательство.

\section{8. ОБОБЩЕНИЕ ТЕОРЕМЫ О РСТ-СИММЕТРИИ}

ТЕОРема 16. В теории вайтмановских полей, определенных на пространстве пробньх функиий $S_{\alpha}^{0}\left(\mathbb{R}^{4}\right), \quad \alpha>2$, и удовлетворяющих условию асимптотической коммутативности с нормальной связью спина со статистикой, существует антиунитарный оператор РСТ-симметрии $\Theta$, при действии которого вакуумный вектор остается инвариантным, а поле $\phi_{\iota}$, принадлежащее неприводимому представлению $\left(j_{\iota}, k_{\iota}\right)$ группь $S L(2, \mathbb{C})$, преобразуется по формуле

$$
\Theta \phi_{\iota}(x) \Theta^{-1}=(-1)^{2 j_{\iota}} i^{F_{\iota}} \phi_{\iota}^{*}(-x),
$$

где $F_{\iota}-$ спинорное число поля. 
ДокАЗАТЕЛЬСТво. Как известно, в терминах вайтмановских функций необходимые и достаточные условия существования оператора $\Theta$ имеют вид

$$
\mathcal{W}_{\iota_{1} \ldots \iota_{n}}\left(x_{1}, \ldots, x_{n}\right)=(-1)^{2 J} i^{F} \mathcal{W}_{\iota_{n} \ldots \iota_{1}}\left(-x_{n}, \ldots,-x_{1}\right)
$$

где $J=j_{\iota_{1}}+\cdots+j_{\iota_{n}}, F=F_{\iota_{1}}+\cdots+F_{\iota_{n}}$ - число полей с полуцелым спином в наборе $\phi_{\iota_{1}}, \ldots, \phi_{\iota_{n}}$. Запишем разность левой и правой частей формулы (44) следующим образом:

$$
\begin{aligned}
& {\left[\mathcal{W}_{\iota_{1} \ldots \iota_{n}}\left(x_{1}, \ldots, x_{n}\right)-(-1)^{2 J} \mathcal{W}_{\iota_{1} \ldots \iota_{n}}\left(-x_{1}, \ldots,-x_{n}\right)\right]+} \\
& \quad+(-1)^{2 J}\left[\mathcal{W}_{\iota_{1} \ldots \iota_{n}}\left(-x_{1}, \ldots,-x_{n}\right)-i^{F} \mathcal{W}_{\iota_{n} \ldots \iota_{1}}\left(-x_{n}, \ldots,-x_{1}\right)\right]
\end{aligned}
$$

При условии асимптотической коммутативности выражение во вторых квадратных скобках сосредоточено на конусе $\bigcup_{k<l}\left\{x \in \mathbb{R}^{4 n}:\left(x_{k}-x_{l}\right)^{2} \geqslant 0\right\}$, который содержится в дополнении к конусу Йоста, поскольку точки Йоста вполне пространственноподобны. Согласно теореме 10 конус $\complement \mathcal{J}_{n}$ является несущим и для функционала в первых квадратных скобках выражения (45). В импульсном представлении обе входящие в (44) обобщенные функции сосредоточены в остром конусе

$$
\left\{p \in \mathbb{R}^{4 n}: \sum_{k=1}^{n} p_{k}=0, \quad \sum_{k=1}^{l} p_{k} \in \overline{\mathbb{V}}_{+}, \quad l=1, \ldots, n-1\right\}
$$

Значит, равенство (44) соблюдается тождественно по теореме 6. Оператор $\Theta$ теперь строится стандартным образом. Сначала определяем его на векторах, получаемых применением к вакууму мономов по полям, полагая

$$
\Theta \Psi_{0}=\Psi_{0}, \quad \Theta \phi_{\iota_{1}}\left(f_{1}\right) \ldots \phi_{\iota_{n}}\left(f_{n}\right) \Psi_{0}=(-1)^{2 J} i^{F} \phi_{\iota_{1}}^{*}\left(\tilde{f}_{1}\right) \ldots \phi_{\iota_{n}}^{*}\left(\tilde{f}_{n}\right) \Psi_{0}
$$

где $\tilde{f}(x)=\bar{f}(-x)$. Легко проверить, что это определение корректно. Именно, при учете того, что поле $\phi_{\iota}^{*}$ преобразуется по сопряженному представлению $\left(k_{\iota}, j_{\iota}\right)$, из формулы (44) для векторов такого вида вытекает равенство $\langle\Theta \Phi, \Theta \Psi\rangle=\overline{\langle\Phi, \Psi\rangle}$. Поэтому, если один и тот же вектор $\Psi$ порождается разными мономами $M_{1}\left(f_{1}\right), M_{2}\left(f_{2}\right)$, то скалярное произведение $\left\langle\Theta M_{1} \Psi_{0}, \Theta M_{2} \Psi_{0}\right\rangle$ равно квадрату длины каждого из векторов $\Theta M_{1} \Psi_{0}, \Theta M_{2} \Psi_{0}$, т.е. эти векторы совпадают. Аналогичным образом, если $\Psi=\Psi_{1}+$ $\Psi_{2}$, где все векторы порождены действием мономов на $\Psi_{0}$, то $\Theta \Psi=\Theta \Psi_{1}+\Theta \Psi_{2}$. Значит, оператор $\Theta$ допускает продолжение по антилинейности на $D_{0}$. Дальнейшее продолжение по непрерывности дает антиунитарный оператор в $\mathcal{H}$.

Теореме 16 можно придать более сильную формулировку, если ввести аналог условия слабой локальной коммутативности Йоста-Дайсона. 
ОПреДЕЛЕНИЕ 2. Пусть поля $\left\{\phi_{\iota}\right\}$ определены на пространстве $S_{\alpha}^{0}\left(\mathbb{R}^{4}\right)$. Мы говорим, что они удовлетворяют условию слабой асимптотической коммутативности, если для любого набора индексов $\iota_{1}, \ldots, \iota_{n}$ функционал

$$
\left\langle\Psi_{0}, \phi_{\iota_{1}}\left(x_{1}\right) \ldots \phi_{\iota_{n}}\left(x_{n}\right) \Psi_{0}\right\rangle-i^{F}\left\langle\Psi_{0}, \phi_{\iota_{n}}\left(x_{n}\right) \ldots \phi_{\iota_{1}}\left(x_{1}\right) \Psi_{0}\right\rangle
$$

сосредоточен на конусе $\complement \mathcal{J}_{n}$, дополнительном к конусу Йоста.

Из сказанного выше ясно, что это условие эквивалентно равенству (44), т.е. справедливо следуюшее утверждение.

ТЕОрема 17. Теория поля с пробными функциями в пространстве $S_{\alpha}^{0}\left(\mathbb{R}^{4}\right)$, $\alpha>2$, в которой выполнены все аксиомы Вайтмана, за исключением локальности, является РСТ-инвариантной тогда и только тогда, когда соблюдается условие слабой асимптотической коммутативности.

Более того, согласно теореме 6 теория поля обладает $P C T$-симметрией и в том случае, если разность (46) сосредоточена в дополнении к конусу, порождаемому любой сколь угодно малой вешественной окрестностью какой-либо точки Йоста. Наше рассмотрение показывает также, что для условия слабой локальности справедлив своего рода аналог теоремы о глобальной природе локальной коммутативности. В наиболее сильной формулировке, принадлежащей Борхерсу и Полмейеру [27], эта теорема утверждает, что в теории (скалярного) поля умеренного роста ограничение вида

$$
\left|\left\langle\Psi_{0},\left[\phi\left(x_{1}\right), \phi\left(x_{2}\right)\right] \phi\left(x_{3}\right) \ldots \phi\left(x_{n}\right) \Psi_{0}\right\rangle\right| \leqslant C_{n} \exp \left\{-\gamma\left|\left(x_{1}-x_{2}\right)^{2}\right|^{\frac{p}{2}}\right\} \quad(p>1)
$$

на поведение коммутатора в тех точках $\left(x_{1}, x_{2}, x_{3}, \ldots, x_{n}\right)$, которые принадлежат конусу $\mathcal{J}_{n}$ вместе с $\left(x_{2}, x_{1}, x_{3}, \ldots, x_{n}\right)$, влечет строгую локальную коммутативность. Аналогичным образом, если все поля $\phi_{\iota}$ определены на пространстве Шварца $S$ и функционал (46) убывает в конусе $\mathcal{J}_{n}$ по экспоненциальному закону типа $(47)$, то он равен нулю всюду в этом конусе. Действительно, используя разложение единицы и учитывая замечание раздела 3 , мы видим, что тогда сужение функционала (46) на пространство $S_{\alpha}^{0}\left(\mathbb{R}^{4 n}\right)$ имеет $\complement \mathcal{J}_{n}$ своим несушим конусом. Значит, теория обладает $P C T$-симметрией и обязана быть слаболокальной в силу обычной $P C T$-теоремы [2-4].

\section{9. ЗАКЛЮЧИТЕЛЬНЫЕ ЗАМЕЧАНИЯ}

Основной результат данной работы состоит в строгом доказательстве сохранения нормальной связи спина со статистикой и $P C T$-инвариантности при замене аксиомы микропричинности условием убывания (анти)коммутаторов полей при пространственноподобной раздвижке аргументов быстрее любой линейной экспоненты, корректно сформулированным в терминах теории аналитических функционалов. Отсутствие каких-либо экспериментальных указаний на нарушение этих двух фундаментальных 
свойств квантовой физики принято считать аргументом в пользу локальности взаимодействия. Мы видим, что они имеют более глубокие корни в математической структуре квантовой теории поля и носят, в сущности, асимптотический характер. Полученное обобшение теорем о связи спина со статистикой и РCT-симметрии является максимально возможным в том смысле, что при допущении экспоненциального убывания с конечным показателем становится допустимым, как отмечал еще Паули [28], квантование скалярного поля посредством аномального соотношения $[\phi(x), \phi(y)]_{+}=\Delta^{(1)}(x-y)$, где $\Delta^{(1)}$ - четное решение свободного уравнения с поведением $\sim \exp (-m|\mathbf{x}-\mathbf{y}|)$ на пространственной бесконечности.

В связи со сделанным выше замечанием относительно теоремы о глобальной природе локальности следует подчеркнуть, что в применении к полям умеренного роста (т.е. к их сужению на $\left.S_{\alpha}^{0}\left(\mathbb{R}^{4}\right)\right)$ условие асимптотической коммутативности не сводится к наивному ограничению (47) и не влечет строгую локальную коммутативность. Строго говоря, это условие означает быстрое убывание не самого коммутатора, а результата его сглаживания с соответствующими пробными функциями (подробнее см. [1]), что кажется более оправданным требованием с физической точки зрения.

Перечисленные в разделе 2 теоремы о несущих конусах аналитических функционалов были установлены в работах [10-13] с целью применения к ковариантному квантованию калибровочных моделей, где сингулярности имеют инфракрасное происхождение и пространства $S_{\alpha}^{\beta}$ с индексом $\beta<1$ служат естественной функциональной областью определения полей в импульсном представлении при их операторной реализации в ковариантной калибровке обшего вида [29]. Этот формализм дает, в частности, простой и общий метод построения нормально упорядоченных целых функций свободного поля с индефинитной метрикой в пространствах Гильберта-Фока-Крейна [30]. В данной работе эффективность развитой техники показана на примере решения классических задач нелокальной теории поля. Она позволяет фактически завершить распространение вайтмановского аксиоматического подхода на нелокальные поля с произвольным высокоэнергетическим поведением.

Благодарности. Автор признателен проф. В.Я. Файнбергу за полезное обсуждение. Он благодарен также за финансовую поддержку, оказанную работе грантами РФФИ № 96-01-00105 и ИНТАС № 96-0308.

\section{Список литературы}

[1] V. Ya. Fainberg, M. A. Soloviev. ТМФ. 1992. V. 93. Р. 514.

[2] Н. Н. Боголюбов, А. А. Логунов, А. И. Оксак, И. Т. Тодоров. Общие принципы квантовой теории поля. М.: Наука, 1987.

[3] Р. Йост. Общая теория квантованных полей. М.: Мир, 1967.

[4] P. Cтритер, A. Вайтман. РCT, спин и статистика и все такое. М.: Наука, 1966.

[5] W. Lücke. Commun. Math. Phys. 1979. V. 65. P. 77.

[6] W. Lücke. Acta Phys. Austriaca. 1984. V. 55. P. 213.

[7] W. Lücke. J. Math. Phys. 1986. V. 27. P. 1901.

[8] M. A. Soloviev. J. Math. Phys. 1998. V. 39. P. 2635

[9] М. А. Соловьев. Письма в ЖЭТФ. 1998. Т. 67. С. 586.

[10] M. A. Soloviev. Lett. Math. Phys. 1995. V. 33. P. 49. 
[11] М. А. Соловьев. ТМФ. 1995. Т. 105. С. 405.

[12] M. A. Soloviev. Commun. Math. Phys. 1997. V. 184. P. 579.

[13] M.A. Soloviev. Carrier cones of the Fourier transforms of ultradistributions. Preprint FIAN/TD/8-99. М.: ФИАН, 1999.

[14] В.Я. Файнберг. О квантовых теориях с неполиномиальным ростом матричных элементов. В сб.: Проблемы теоретической физики. Ред. В.И. Ритус, Е. Л. Фейнберг и др. М.: Наука, 1972. C. 119.

[15] Г. В. Ефимов. Нелокальные взаимодействия квантованных полей. М.: Наука, 1977.

[16] Г. В. Ефимов. Проблемы квантовой теории нелокальных взаимодействий. М.: Наука, 1985.

[17] М. А. Соловьев. Труды ФИАН. 1993. Т. 209. С. 121.

[18] J. Bümmerstede, W. Lücke. Commun. Math. Phys. 1974. V. 37. P. 121.

[19] V. Ya. Fainberg, A. V. Marshakov. Phys. Lett. B. 1988. V. 211. P. 82.

[20] A.S. Wightman. Adv. Math. Suppl. Stud. 1981. V. 7B. P. 769.

[21] И. М. Гельфанд, Г. Е. Шилов. Обобщенные функции. Т. 2. М.: Физматгиз, 1958.

[22] П. Шапира. Теория гиперфункций. М.: Мир, 1972.

[23] Л. Хёрмандер. Анализ линейных дифференциальных операторов с частными производными. Т. 1. М.: Мир, 1986.

[24] H. Komatsu. J. Fac. Sci. Univ. Tokyo, Sect. 1A, Math. 1973. V. 20. P. 25.

[25] Х. Шефер. Топологические векторные пространства. М.: Мир, 1971.

[26] М. А. Соловьев. ТМФ. 1973. Т. 15. С. 3.

[27] H. J. Borchers, K. Pohlmeyer. Commun. Math. Phys. 1968. V. 8. P. 269.

[28] В. Паули. Принцип запрета, группа Лоренца, отражение пространства, времени и заряда. В сб.: Нильс Бор и развитие физики. Ред. В. Паули при участии Л. Розенфельда и В. Вайскопфа. М.: ИЛ, 1958. С. 46.

[29] U. Moschella, F. Strocchi. Lett. Math. Phys. 1992. V. 24. P. 103.

[30] M. A. Soloviev. Lett. Math. Phys. 1997. V. 41. P. 265.

Поступила в редакцию 18.XII.1998 г. 